\title{
Negative pressure wound therapy in patients with wounds healing by secondary intention: a systematic review and meta- analysis of randomised controlled trials
}

Yvonne Zens $^{1 *}$ (D), Michael Barth, Heiner C. Bucher ${ }^{2}$, Katrin Dreck', Moritz Felsch¹, Wolfram Groß¹, Thomas Jaschinski ${ }^{1}$, Heike Kölsch ${ }^{1}$, Mandy Kromp ${ }^{1}$, Inga Overesch', Stefan Sauerland ${ }^{1}$ and Sven Gregor ${ }^{3}$

\begin{abstract}
Background: Negative pressure wound therapy (NPWT) is a widely used method of wound treatment. We performed a systematic review of randomised controlled trials (RCTs) comparing the patient-relevant benefits and harms of NPWT with standard wound therapy (SWT) in patients with wounds healing by secondary intention.

Methods: We searched for RCTs in MEDLINE, Embase, the Cochrane Central Register of Controlled Trials, and study registries (last search: July 2018) and screened reference lists of relevant systematic reviews and health technology assessments. Manufacturers and investigators were asked to provide unpublished data. Eligible studies investigated at least one patient-relevant outcome (e.g. wound closure). We assessed publication bias and, if feasible, performed meta-analyses, grading the results into different categories (hint, indication or proof of a greater benefit or harm).

Results: We identified 48 eligible studies of generally low quality with evaluable data for 4315 patients and 30 eligible studies with missing data for at least 1386 patients. Due to potential publication bias (proportion of inaccessible data, 24\%), we downgraded our conclusions. A meta-analysis of all wound healing data showed a significant effect in favour of NPWT (OR 1.56, 95\% Cl 1.15 to 2.13, $p=0.008$ ). As further analyses of different definitions of wound closure did not contradict that analysis, we inferred an indication of a greater benefit of NPWT. A meta-analysis of hospital stay (in days) showed a significant difference in favour of NPWT (MD - 4.78, 95\% $\mathrm{Cl}-7.79$ to $-1.76, p=0.005$ ). As further analyses of different definitions of hospital stay/readmission did not contradict that analysis, we inferred an indication of a greater benefit of NPWT. There was neither proof (nor indication nor hint) of greater benefit or harm of NPWT for other patient-relevant outcomes such as mortality and adverse events.

\footnotetext{
* Correspondence: Yvonne.Zens@iqwig.de

'Institute for Quality and Efficiency in Health Care (IQWiG), Im Mediapark 8, 50670 Cologne, Germany

Full list of author information is available at the end of the article
}

(C) The Author(s). 2020 Open Access This article is licensed under a Creative Commons Attribution 4.0 International License, which permits use, sharing, adaptation, distribution and reproduction in any medium or format, as long as you give appropriate credit to the original author(s) and the source, provide a link to the Creative Commons licence, and indicate if changes were made. The images or other third party material in this article are included in the article's Creative Commons. licence, unless indicated otherwise in a credit line to the material. If material is not included in the article's Creative Commons licence and your intended use is not permitted by statutory regulation or exceeds the permitted use, you will need to obtain permission directly from the copyright holder. To view a copy of this licence, visit http://creativecommons.org/licenses/by/4.0/ The Creative Commons Public Domain Dedication waiver (http://creativecommons.org/publicdomain/zero/1.0/) applies to the data made available in this article, unless otherwise stated in a credit line to the data. 
(Continued from previous page)

Conclusions: In summary, low-quality data indicate a greater benefit of NPWT versus SWT for wound closure in patients with wounds healing by secondary intention. The length of hospital stay is also shortened. The data show no advantages or disadvantages of NPWT for other patient-relevant outcomes. Publication bias is an important problem in studies on NPWT, underlining that all clinical studies need to be fully reported.

Keywords: Negative-pressure wound therapy, Wound healing, Benefit assessment, Systematic review, Publication bias

\section{Background}

Chronic wounds affect about $1 \%$ of the population in Western industrialised countries, with much higher rates in inpatient settings, and pose a serious risk to patients' health and quality of life [1-4]. Negative pressure wound therapy (NPWT), also called vacuum-assisted wound closure, was introduced into clinical practice in the early 1990s. With this technique, an open-cell foam dressing is placed into the wound cavity and a controlled subatmospheric pressure is applied to suck fluid from the wound, with the intention of improving wound healing [5]. In the past decades, the use of NPWT has increased considerably and it is currently applied across the world in both inpatient and outpatient settings for various surgical indications. Although multiple clinical benefits have been described, most clinical studies or evidence syntheses have failed to prove statistically significant or clinically relevant benefits versus standard wound therapy (SWT). For instance, in 2006, the German Institute for Quality and Efficiency in Health Care (Institut für Qualität und Wirtschaftlichkeit im Gesundheitswesen, IQWiG) conducted a health technology assessment (HTA) of NPWT studies [6] followed by a rapid report in 2007 [7] and found that "although there is some indication that NPWT may improve wound healing, the body of evidence available is insufficient to clearly prove an additional clinical benefit of NPWT. The large number of prematurely terminated and unpublished trials is the reason for concern" [8]. The IQWiG reports contained only a few small studies (all conducted in Western industrialised countries), all of poor methodological quality. In the meantime, considerably more evidence has accumulated on NPWT from randomised controlled trials (RCTs) conducted in multiple surgical indications and settings.

The aim of this systematic review of RCTs was therefore to assess the patient-relevant benefits and harms of NPWT versus SWT. Due to numerous and changing surgical indications and further developments in technology, our analysis considered all wounds healing by secondary intention.

\section{Methods}

\section{Protocol and methodological approach}

Our review formed part of a German-language HTA of the benefits and harms of NPWT in patients with wounds healing by secondary intention published by IQWiG in 2019. The full (German-language) protocol and report (Commission No. N17-01A) are available on the Institute's website [9]. Both the preliminary protocol and the preliminary report underwent public commenting procedures. IQWiG's responsibilities and methodological approach are described in its methods paper [10]. Only completed studies were used, so there was no need for ethical approval and patient consent. We adhered to the PRISMA statement [11] throughout this manuscript.

\section{Eligibility criteria}

We included both published and previously unpublished RCTs comparing NPWT for wounds healing by secondary intention with any kind of SWT and investigating at least one predefined patient-relevant outcome. In this context, the term "patient-relevant" refers to "how a patient feels, functions or survives" [12]. The detailed eligibility criteria are presented in Table 1

\section{Search strategy and study selection}

This systematic review is based on two previous HTA reports of IQWiG [6, 7]. We conducted an update search for the period not covered by these reports (from 2006 onwards). We searched the following bibliographic databases: MEDLINE, Embase, the Cochrane Central Register of Controlled Trials, the Cochrane Database of Systematic Reviews, and the Health Technology Assessment Database. The peer-reviewed search strategy included a combination of subject headings and free text with terms such as "negative pressure wound therapy" and "vacuum-assisted closure" (see Additional file 1 for the full search strategy). In addition, we searched ClinicalTrials.gov and the International Clinical Trials Registry Platform Search Portal. The last search was run on July 24, 2018. The reference lists of relevant systematic reviews and HTA reports published between 2013 and 2018 were scrutinised to identify further studies. In order to obtain the most complete data set possible, we also asked NPWT manufacturers to supply unpublished studies and additional unpublished data from published studies (see Additional file 1 for the full list of manufacturers). 
Table 1 Eligibility criteria for studies included

\begin{tabular}{|c|c|}
\hline Population & $\begin{array}{l}\text { - Patients with wounds with intended secondary healing } \\
\text { - Any healthcare setting }\end{array}$ \\
\hline Study intervention & $\begin{array}{l}\text { - Negative pressure wound therapy } \\
\text { o No restrictions with regard to the use of commercial and/or custom-made devices } \\
\text { o Type of further treatment, in particular indication for surgical wound closure, comparable to control intervention }\end{array}$ \\
\hline Control intervention & $\begin{array}{l}\text { - Standard wound therapy } \\
\text { o Type of further treatment, in particular indication for surgical wound closure, comparable to study intervention }\end{array}$ \\
\hline $\begin{array}{l}\text { Patient-relevant } \\
\text { outcomes }\end{array}$ & $\begin{array}{l}\text { - Mortality } \\
\text { - Wound closure } \\
\text { - Adverse events } \\
\text { - Amputation } \\
\text { - Pain } \\
\text { - Length of hospital stay and/or readmission to the hospital } \\
\text { - Health-related quality of life } \\
\text { - Physical function } \\
\text { - Dependence on outside help or need for care }\end{array}$ \\
\hline Study design & $\begin{array}{l}\text { - Randomised controlled trials } \\
\text { o Data of studies with fewer than } 10 \text { patients were excluded from the assessment. For transparency reasons those studies } \\
\text { meeting the other eligibility criteria were included in the initial pool of relevant studies. }\end{array}$ \\
\hline Publication type & $\begin{array}{l}\text { - Availability of a full-text document (e.g. journal article or clinical study report, CSR) } \\
\text { - No restrictions applied for the date of publication }\end{array}$ \\
\hline Timing & - No restrictions \\
\hline $\begin{array}{l}\text { Language of } \\
\text { publication }\end{array}$ & - Any language if English titles and abstracts were available and indicated potential relevance \\
\hline
\end{tabular}

As a prerequisite for the use of unpublished data, IQWiG asked the manufacturers to sign an agreement requiring (1) the submission of a list of all sponsored published and unpublished studies investigating NPWT and (2) the submission of CONSORT-compliant documents (in general the complete clinical study reports, CSRs) on all relevant studies selected by IQWiG. This procedure was required to avoid bias through the selective provision of data. Furthermore, we contacted the investigators responsible for investigator-initiated trials (IITs) to obtain the current study status or even data from potentially completed studies identified in study registries. In addition, persons and parties who had submitted comments on the preliminary version of the IQWiG report in the written public hearing were asked to provide any additional relevant studies.

Two reviewers independently screened titles and abstracts of the retrieved citations to identify potentially eligible primary and secondary publications. The full texts of these articles were obtained and independently evaluated by the same reviewers. All documents retrieved from non-bibliographical sources were also screened for eligibility or relevant information on studies. Disagreements were resolved by consensus.

Literature searching and study selection were done in parallel for two HTA reports, one on NPWT in patients with wounds healing by primary intention and one on NPWT in patients with wounds healing by secondary intention. The results of the HTA report on wounds healing by primary intention will be reported separately.

\section{Data extraction}

The individual steps of the data extraction and risk-ofbias assessment procedures were always conducted by one person and checked by another; disagreements were resolved by consensus. Details of the studies were extracted using standardised tables.

We extracted information on:

(1) Study characteristics, including the study design, length of follow-up, sample size, location, number of centres and period in which the study had been conducted.

(2) Characteristics of the study participants, including inclusion and exclusion criteria, age, sex, wound characteristics at baseline, time since wound occurrence and dropout rate.

(3) Characteristics of the test and control interventions, including treatment regimens and concomitant treatments.

(4) Outcomes and type of outcome measures: outcomes as presented above; we did not limit the types of measures for a specific outcome, but rather analysed all measures used (e.g. wound healing (yes/ no), time to wound healing).

(5) Risk-of-bias items (see below). Information and data from publications were supplemented by publicly available results data from study registries and unpublished CSRs provided by manufacturers or IIT investigators. 


\section{Assessment of risk of bias in individual studies}

Using the IQWiG methods, we assessed the risk of bias (high or low) on the study and outcome level [10]. Because of the large number of studies, we conducted a stepwise assessment: if the generation of a randomisation sequence and/or the allocation concealment were inadequate, we assigned a high risk of bias to the study. If we did not, the following items were assessed at study level across outcomes: blinding of patients and treating staff, reporting of all relevant outcomes independent of results and other aspects, such as differences in the length of follow-up.

A high risk of bias on the study level generally led to a high risk of bias on the outcome level. Otherwise, the following outcome-specific items were assessed: blinding of outcome assessors, appropriate application of the intention-to-treat principle, reporting of individual outcomes independent of results and other aspects.

Using the IQWiG methods, we graded the results of the (meta-)analysis into different categories: proof, indication and hint (or neither proof, nor indication nor hint) of a greater benefit of the test intervention. In short, proof of a greater benefit of the test intervention is inferred if a meta-analysis of at least 2 studies with a low risk of bias shows a statistically significant effect favouring the test intervention. An indication of a greater benefit is inferred if one single study with a low risk of bias shows a statistically significant effect favouring the test intervention or a meta-analysis of studies with a high risk of bias shows a statistically significant effect favouring the test intervention. A hint of a greater benefit is inferred if a single study with a high risk of bias shows a statistically significant effect favouring the intervention. No proof (or indication or hint) of a greater benefit or harm is inferred if there are no statistically significant differences between the test and control interventions, if relevant heterogeneity exists or if no suitable data are available.

If studies with both a low and a high risk of bias are available for a specific outcome, the studies with a low risk of bias are primarily used to derive proof, an indication or a hint of a greater benefit of NPWT.

In addition to IQWiG methods, we also applied the GRADE approach (Grading of Recommendations Assessment, Development and Evaluation) in order to describe the certainty of the evidence in a widely used framework.

\section{Assessment of publication bias}

Studies missing for analysis were defined as those that fulfilled the eligibility criteria listed above (except for the reporting of at least one patient-relevant outcome), had been completed at least 1 year before the last bibliographic and registry search and were not published.
Studies that had been terminated prematurely or had an unclear study status (no update of study status in the 2 years before our literature search) were also counted as missing, as long as no contrary information was available (e.g. from author inquiries).

We assessed publication bias by comparing the estimated number of patients from missing studies with the number of all patients (from included and missing studies). If the proportion of missing data was $<10 \%$, it was assumed that the impact of bias on the results introduced by the missing data was low and no action was taken. If the proportion of missing data was between 10 and $30 \%$, it was assumed that the impact of bias on the results was high and all conclusions of proof, an indication or a hint of a greater benefit of NPWT were downgraded to an indication, a hint or no hint. If the proportion of missing data was $>30 \%$, it was assumed that the impact of bias on the results was too high to be able to draw robust conclusions and no data analysis was performed.

\section{Data analysis}

If results for different time points were available, the most recent one was used for the analysis, if not stated otherwise. The mean values and standard deviations of continuous variables were derived from the median, minimum and maximum values or the first and third quartile using the method by Wan 2014 [13] or from standard errors or confidence intervals (CI). If no information was available, missing standard deviations were derived from the median values of the standard deviations of all control interventions.

Odds ratios (OR) were calculated to compare dichotomously measured outcomes, mean differences (MD) or Hedges' g were calculated to compare continuously measured outcomes. In most cases, Hedges' g was used to adjust for different wound types, different scales applied for outcome measurements or heterogeneity in the original scale. For all effect estimates, 95\% CI were reported.

If feasible and meaningful, data were pooled by means of meta-analyses. An overall effect was calculated using the Knapp and Hartung method with the Paule-Mandel heterogeneity estimator [14]. If only 2 studies were available, a fixed-effect model with inverse variance [15] was used to combine the study results. We used the betabinomial model [16] to calculate an overall effect estimate to account for studies with no events in both treatment arms (double-zero studies).

If relevant heterogeneity [15] was present $(p<0.05)$, no overall effect estimate was calculated and, if possible, a 95\% prediction interval (PI) [17] was calculated instead.

The results of the meta-analysis were presented in a forest plot. If studies with a low risk of bias showed a 
statistically significant effect, they were presented separately within the same plot.

A $p$ value of $<0.05$ was considered statistically significant.

We also planned subgroup analyses for age, sex, type of indication/wound, type of health care setting, and type of NPWT device.

\section{Results}

\section{Literature search}

A total of 42 eligible studies were identified from 1195 references retrieved from bibliographic databases. Details of the study selection process from bibliographic databases are shown in Fig. 1 . In addition, 20 potentially eligible studies were identified in further sources: 7 studies in the previous IQWIG reports, 5 in the reference lists of other relevant systematic reviews and one in a study registry. Two studies were identified in study registries and became eligible due to data provided by the investigator or manufacturer. Five additional and previously completely unpublished manufacturer-initiated studies finalised the initial study pool (details on data submission by the manufacturers are provided in the current IQWiG report [18]).

The 62 eligible studies (all RCTs) included 14 studies (ActiVac [19], Dwivedi 2016 [20, 21], Eginton 2003 [22], Ford 2002 [23], ISAW [24-26] [27], Joseph 2000 [28], Keskin 2008 [29], Riaz 2010 [30], Sajid 2015 [31], Sun 2007 [32], Vaidhya 2015 [33] and Wanner 2003 [34]) that failed to provide any evaluable data on patientrelevant outcomes. As these studies fulfilled the eligibility criteria and for transparency reasons, they were formally included in the initial study pool but excluded from the analysis. Ultimately, 48 eligible studies with evaluable data on 4315 patients were analysed.

\section{Potential publication bias}

The literature search also identified 45 further studies without any published data on patient-relevant outcomes (of which 12 were planned or ongoing). These studies included 14 terminated, 9 completed studies and 10 studies with an unknown study status-30 out of these 33 studies should already have provided results, as the study had been completed at least 12 months before the search date of the present review. These 30 studies represented missing data of at least 1386 patients; for further details, see Table 2. Compared with the available evaluable data of 4315 patients, this results in a proportion of inaccessible data of $24 \%(1386 / 5701)$ of eligible patients. In consequence, we downgraded the certainty of our conclusions as described in the "Methods" section and refrained from doing subgroup analyses because the results would be hardly interpretable. Detailed documentation of all 45 studies without any published data on patient-relevant outcomes is given in Table 18 of the current IQWiG report [18].

\section{Characteristics of included studies}

Table 3 presents the main characteristics of the 48 studies reporting evaluable results on patient-relevant outcomes. These studies included between 12 and 460 patients and were conducted worldwide between 1998 and 2016. The majority were 2 -arm studies $(n=46)$; one study was 3-armed (Novinščak 2010 [112]) and one was 4 -armed (TOPSKIN [122]). The study design was mostly monocentric $(n=35)$. The majority of studies were performed in an inpatient setting $(n=38)$. In 47 studies, patients were randomised and in one study (Moisidis 2004 [107]) wound halves were randomised. In 45 studies, one wound per patient and in 2 studies (Kakagia 2014 [100] and VAC 2001-06 [132]) at least one wound per patient was analysed.

The 48 studies included covered a wide range of different wounds of various causes: amputation wounds $(n=1$, Liao 2012 [103]), pressure ulcers $(n=$ 2, Ashby 2012 [70], VAC 2001-01 [125]), diabetic foot wounds $(n=6$, Dalla Paola 2010 S-II [86], DiaFu [89], Karatepe 2011 [101], Nain 2011 [111], VAC 2001-07 [135], VAC 2001-08 [142]), diabetic ulcer wounds $(n$ $=1$, Novinščak 2010), foot wounds $(n=1$, Chiang 2017 [83]), fasciotomy wounds due to compartment syndrome $(n=1$, Kakagia 2014), necrotizing fasciitis wounds ( $n=2$, Huang 2006 [98], Xu 2015 [162]), open fractures $(n=7$, Arti 2016 [68], Gupta 2013 [96], Jayakumar 2013 [99], Sibin 2017 [117], VAC 2001-06, Virani 2016 [154], WOLLF [158]), open abdominal wounds $(n=4$, Bee 2008 [73], Correa 2016 [84], Rencüzoğulları 2015 [114], VAC 2002-10 [152]), pilonidal sinus wounds $(n=2$, Banasiewicz 2013 [72], Biter 2014 [74]), open thorax wounds $(n=1$, VAC 2002-09 [150]), traumatic wounds of various causes $(n$ = 3, Llanos 2006 [104], Saaiq 2010 [115], Sinha 2013 [118]), leg ulcer wounds $(n=4$, Leclercq 2016 [102], VAC 2001-02 [128], VAC 2001-03 [130], Vuerstaek 2006 [155]), burns $(n=2$, of which one was in infants, TOPSKIN, Shen 2013 [116]), groyne wounds caused by infection ( $n=1$, Acosta 2013 [65]), and various other wounds due to diseases and/or traumatic or iatrogenic causes $(n=10$, Braakenburg 2006 [76], CE/044/PIC [77], De Laat 2011 [87], Hu 2009 [97], Mody 2008 [105], Moisidis 2004, Mouës 2004 [108], Mohsin 2017 [106], Perez 2010 [113], SWHSI [119]). Comparators were mostly described as standard wound care or standard dressings. If specified, dressings were described as sterilised gauze or moist gauze. Very few sudies provided more detailed information such as alginate, hydrofiber, silver-dressing or polyurethanes. 


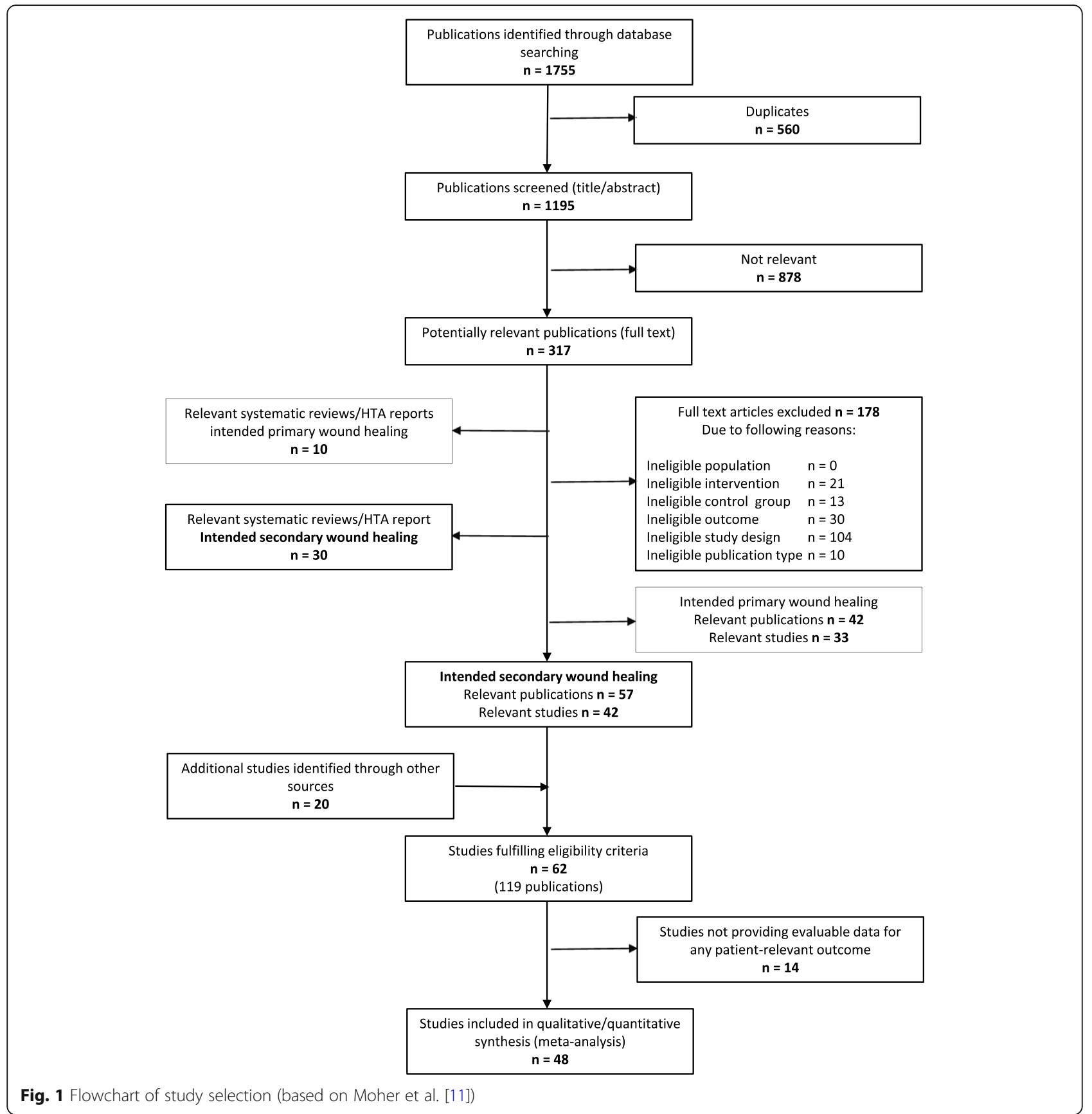

\section{Risk of bias in individual studies}

The risk of bias at the study level was low in 7 (Ashby 2012, DiaFu, Llanos 2006, SWHSI, VAC 200107, Vuerstaek 2006 and WOLLF) out of 48 studies. As shown in Table 4, 41 studies were rated as having a high risk of bias at the study level due to an inadequate description of the randomisation procedure ( $n$ $=20)$ and/or the allocation concealment $(n=37)$, or selective reporting of outcomes $(n=4$; TOPSKIN, VAC 2001-01, VAC 2001-02 and VAC 2001-08). Of the 7 studies rated as having a low risk of bias at the study level, only one also showed a low risk of bias for all outcomes (Llanos 2006). The studies by Ashby 2012 and SWHSI showed a low risk of bias, but not for all of the reported outcomes: pain, wound bleeding and infection (Ashby 2012) as well as adverse events, pain, duration of hospital stay and healthrelated quality of life (SWHSI) were rated as having a high risk of bias. In all other studies, the risk of bias at the outcome level was high. The detailed risk-ofbias assessments for all outcomes in all studies included are presented in Additional file 2. 
Table 2 Unpublished studies considered for assessment of potential publication bias

\begin{tabular}{|c|c|c|c|}
\hline Study & $\begin{array}{l}\text { Sample } \\
\text { size }^{\mathrm{a}}\end{array}$ & $\begin{array}{l}\text { Documents available (e.g. study registry number, study } \\
\text { protocol, CSR) }\end{array}$ & $\begin{array}{l}\text { Recruitment status (estimated study completion } \\
\text { date }^{b} \text { ) }\end{array}$ \\
\hline $\begin{array}{l}\text { ACTR } \\
\text { N12614000056695 }\end{array}$ & 40 & ACTRN12614000056695 [35]/-- & Completed (December 2013) \\
\hline $\begin{array}{l}\text { Adams et al. (2005) } \\
\text { [6] }\end{array}$ & $1^{\mathrm{c}}$ & ---- & Completed (March 2005) ${ }^{d}$ \\
\hline ATEC & 112 & ISRCTN60292377 [36]/-- & Completed (September 2016) \\
\hline B2108R [6] & 120 & NCT00011531 [37]/-- & Completed (December 2001) \\
\hline $\begin{array}{l}\text { CTRI/2018/01/ } \\
011503\end{array}$ & 54 & CTRI/2018/01/011503 [38]/-- & Completed (April 2017) \\
\hline foryou & 48 & ChiCTR-TRC-12002700 [39]/-- & Completed (December 2015) ${ }^{\mathrm{e}}$ \\
\hline VACOTOL-012 & 28 & NCT02102685 [40]/-- & Completed (September 2013) \\
\hline VSD & 119 & ChiCTR-IOR-16008087 [41]/-- & Completed (March 2016) \\
\hline $045-1502-226[6]$ & 30 & NCT00121537 [42]/-- & Terminated (October 2015) ${ }^{\mathrm{e}}$ \\
\hline 2008/2023-31 & $30^{f}$ & NCT01191567 [43]/-- & Terminated (July 2012) \\
\hline ANSM & 36 & NCT02509533 [44]/-- & Terminated (July 2015) \\
\hline Greer et al. (1999) [6] & $16^{9}$ & $--/$ study protocol ${ }^{h}[45]$, raw data ${ }^{h, i}[46]$ & Terminated (November 1999) ${ }^{9}$ \\
\hline HTA012-0801-01 & 184 & NCT00691821 [47]/-- & Terminated (July 2011) ${ }^{\mathrm{e}}$ \\
\hline STOMAVAC & $14^{9}$ & ISRCTN37399763 [48]/-- & Terminated (December 2014) \\
\hline U1111-1132-0768 & 30 & ACTRN12612000702819 [49]/-- & Terminated $^{f}(\text { n.s. })^{J}$ \\
\hline U1111-1133-5694 & $0^{f}$ & ACTRN12612000885897 [50]/-- & Terminated $^{f}$ (n.s.) \\
\hline U1111-1162-0654 & $16^{\mathrm{f}}$ & ACTRN12614001068651 [51]/-- & Terminated $(n . s .)^{J}$ \\
\hline VAC 2001-00 [6] & 46 & -- /study protocol' [52], CSR $\mathrm{CR}^{\mathrm{h}, \mathrm{k}}[53]$ & Terminated $^{g}(\text { n.s. })^{J}$ \\
\hline VAC 2006-19 & $19^{9}$ & NCT00837096 [54]/study protocol ${ }^{\mathrm{h}}[55]$ & Terminated (October 2013) ${ }^{\mathrm{e}}$ \\
\hline VAC TRIAL & 9 & ACTRN12606000384550 [56]/study protocol ${ }^{h}[57]$ & Terminated (September 2005) \\
\hline 2015046 & 80 & NCT02374528 [58]/-- & Unknown (April 2016) \\
\hline $382094-2$ & 30 & NCT01857128 [59]/-- & Unknown ( December 2014) \\
\hline $\begin{array}{l}\text { ACTR } \\
\text { N12609000149268 }\end{array}$ & 60 & ACTRN12609000149268 [60]/-- & Unknown' (n.s.) ${ }^{\jmath}$ \\
\hline $\begin{array}{l}\text { ACTR } \\
\text { N12609000995279 }\end{array}$ & 100 & ACTRN12609000995279 [61]/-- & Unknown' (n.s.) ${ }^{\jmath}$ \\
\hline $\begin{array}{l}\text { CTRI/2014/02/ } \\
004390\end{array}$ & 40 & CTRI/2014/02/004390 [62]/-- & Unknown' (n.s.) ${ }^{\jmath}$ \\
\hline Foo et al. (2004) [6] & $--^{m}$ & $--/-$ & Unknown ${ }^{d}$ (n.s.) \\
\hline $\begin{array}{l}\text { Gupta et al. (2001) } \\
\text { [6] }\end{array}$ & $1^{c}$ & ---- & Unknown ${ }^{\text {(n.s.) }}$ \\
\hline ITIQ002A & 90 & NCT01734109 [63]/-- & Unknown (March 2014) \\
\hline McCarthy M 2005 & $1^{\mathrm{c}}$ & $--/-$ & Unknown (n.s.) $)^{\jmath}$ \\
\hline NPWTVsGPA & 32 & NCT02314468 [64]/-- & Unknown (October 2016) \\
\hline
\end{tabular}

CSR clinical study report, $n$.s. not specified

a Number of patients counted as missing; according to study registry information, if not stated otherwise

${ }^{\mathrm{b}}$ According to study registry information, if not stated otherwise

'Not known; $N=1$ used as a placeholder

${ }^{\mathrm{d}}$ According to status of previous HTA report N04-03; no further information available

${ }^{\mathrm{e}}$ Date of last study registry update; study may have been completed/terminated for a longer period of time

fAccording to author's reply

${ }^{9}$ According to a manufacturer's reply

${ }^{\mathrm{h}}$ Not publically available

'Raw data provided by a manufacturer; refer to less than $70 \%$ of included patients; no data for planned patient-relevant outcomes included

${ }^{\mathrm{j} A c c o r d i n g}$ to the available information study, study should have been completed/terminated for more than 12 months

${ }^{\mathrm{k}} \mathrm{It}$ is not possible to certainly assign the CSR provided by the manufacturer to the study under investigation. Furthermore, several pages had

been deleted

"Classification as "unknown" as the status had not been updated within the 2 years before our literature search

mAccording to the previous HTA report N04-03 [6], change in wound surface should have been investigated. This outcome does not represent a patient-relevant outcome. The study was not further taken into account 
Table 3 Characteristics of included studies

\begin{tabular}{|c|c|c|c|c|c|c|c|c|}
\hline Study & $\begin{array}{l}\text { Study } \\
\text { design }\end{array}$ & $\begin{array}{l}N \\
\text { participants } \\
\text { randomised }\end{array}$ & $\begin{array}{l}\text { Duration of } \\
\text { active treatment } \\
\text { (intervention } \\
\text { group) }\end{array}$ & $\begin{array}{l}\text { Study } \\
\text { duration } \\
\text { (including } \\
\text { length of } \\
\text { follow-up) }\end{array}$ & Setting & $\begin{array}{l}\text { Location and } \\
\text { study period }\end{array}$ & $\begin{array}{l}\text { Relevant } \\
\text { outcomes }\end{array}$ & Indication/wound type \\
\hline $\begin{array}{l}\text { Acosta et al. } \\
\text { (2013) [65-67] }\end{array}$ & $\begin{array}{l}\text { RCT } \\
\text { Blinding } \\
\text { not } \\
\text { specified } \\
\text { Single } \\
\text { centre }\end{array}$ & 20 & Not specified & $\begin{array}{l}\text { Until } \\
\text { complete } \\
\text { epithelization } \\
\text { of the skin }\end{array}$ & $\begin{array}{l}\text { Inpatient } \\
\text { with } \\
\text { outpatient } \\
\text { continuation }\end{array}$ & $\begin{array}{l}\text { Sweden } \\
\text { February } \\
2007-\text { April } \\
2012\end{array}$ & $\begin{array}{l}\text { Mortality } \\
\text { Wound closure } \\
\text { Adverse events } \\
\text { Amputation } \\
\text { Hospital stay } \\
\text { and } \\
\text { readmission }\end{array}$ & $\begin{array}{l}\text { Acute open wounds } \\
\text { Deep peri-vascular groyne infec- } \\
\text { tions (Szilagyi grade III) }\end{array}$ \\
\hline $\begin{array}{l}\text { Arti et al. } \\
(2016)[68,69]\end{array}$ & $\begin{array}{l}\text { RCT } \\
\text { Blinding } \\
\text { not } \\
\text { specified } \\
\text { Single } \\
\text { centre }\end{array}$ & 90 & $\begin{array}{l}\text { Generally 10-14 } \\
\text { days }\end{array}$ & 1 month & Inpatient & $\begin{array}{l}\text { Iran } \\
\text { February } \\
\text { 2013-March } \\
2015\end{array}$ & $\begin{array}{l}\text { Wound closure } \\
\text { Adverse events }\end{array}$ & $\begin{array}{l}\text { Acute open wounds } \\
\text { Open fracture wound type IIIb } \\
\text { based on Gustilo-Anderson } \\
\text { classification }\end{array}$ \\
\hline $\begin{array}{l}\text { Ashby et al. } \\
(2012)[70,71]\end{array}$ & $\begin{array}{l}\text { RCT } \\
\text { Outcome- } \\
\text { assessor } \\
\text { blinded } \\
\text { Single } \\
\text { centre }\end{array}$ & 12 & $\begin{array}{l}\text { According to the } \\
\text { requirements of } \\
\text { the nursing staff }\end{array}$ & 6 months & $\begin{array}{l}\text { Inpatient, } \\
\text { nursing } \\
\text { home and } \\
\text { patient's } \\
\text { home }\end{array}$ & $\begin{array}{l}\text { UK } \\
\text { September } \\
\text { 2008-August } \\
2009\end{array}$ & $\begin{array}{l}\text { Mortality } \\
\text { Wound closure } \\
\text { Adverse events } \\
\text { Pain }\end{array}$ & $\begin{array}{l}\text { Chronic open wounds } \\
\text { Grade III/N pressure ulcers } \\
\text { according to the European } \\
\text { Pressure Ulcer Advisory Panel } \\
\text { Grading System }\end{array}$ \\
\hline $\begin{array}{l}\text { Banasiewicz } \\
\text { et al. (2013) } \\
{[72]}\end{array}$ & $\begin{array}{l}\text { RCT } \\
\text { Blinding } \\
\text { not } \\
\text { Specified } \\
\text { Single } \\
\text { centre }\end{array}$ & 19 & Not specified & $\begin{array}{l}\text { Until the } \\
\text { wounds } \\
\text { healed to } \\
\text { restore } \\
\text { normal } \\
\text { activity }\end{array}$ & Outpatient & $\begin{array}{l}\text { Poland } \\
2012\end{array}$ & $\begin{array}{l}\text { Pain } \\
\text { Physical } \\
\text { function }\end{array}$ & $\begin{array}{l}\text { Acute open wounds } \\
\text { Pilonidal sinus (primary/ } \\
\text { recurrent) }\end{array}$ \\
\hline $\begin{array}{l}\text { Bee et al. } \\
\text { (2008) [73] }\end{array}$ & $\begin{array}{l}\text { RCT } \\
\text { Blinding } \\
\text { not } \\
\text { Specified } \\
\text { Single } \\
\text { centre }\end{array}$ & 51 & maximum 9 days & Not specified & Inpatient & $\begin{array}{l}\text { USA } \\
\text { April } 2003- \\
\text { July } 2007\end{array}$ & $\begin{array}{l}\text { Mortality } \\
\text { Adverse events }\end{array}$ & $\begin{array}{l}\text { Acute open wounds } \\
\text { Temporary abdominal closure } \\
\text { after damage control } \\
\text { laparotomy, massive visceral } \\
\text { oedema and planned } \\
\text { reexploration }\end{array}$ \\
\hline $\begin{array}{l}\text { Biter et al. } \\
(2014)[74,75]\end{array}$ & $\begin{array}{l}\text { RCT } \\
\text { Unblinded } \\
\text { Single } \\
\text { centre }\end{array}$ & 49 & 14 days & $\begin{array}{l}6 \text { months } \\
\text { after wound } \\
\text { closure }\end{array}$ & Outpatient & $\begin{array}{l}\text { Netherlands } \\
\text { October } \\
\text { 2009-May } \\
2012\end{array}$ & $\begin{array}{l}\text { Wound closure } \\
\text { Adverse events } \\
\text { Pain } \\
\text { Physical } \\
\text { function }\end{array}$ & $\begin{array}{l}\text { Acute open wounds } \\
\text { Symptomatic pilonidal sinus } \\
\text { with or without a previous } \\
\text { abscess of the sinus }\end{array}$ \\
\hline $\begin{array}{l}\text { Braakenburg } \\
\text { et al. (2006) } \\
{[76]}\end{array}$ & $\begin{array}{l}\text { RCT } \\
\text { Blinding } \\
\text { not } \\
\text { Specified } \\
\text { Single } \\
\text { centre }\end{array}$ & 64 & Not specified & Until 80 days & Inpatient & $\begin{array}{l}\text { Netherlands } \\
\text { March 2002- } \\
\text { May } 2004\end{array}$ & $\begin{array}{l}\text { Mortality } \\
\text { Wound closure } \\
\text { Adverse events } \\
\text { Amputation }\end{array}$ & $\begin{array}{l}\text { Acute, subacute and chronic } \\
\text { wounds } \\
\text { Any type of wound }\end{array}$ \\
\hline $\begin{array}{l}\text { CE/044/PIC } \\
{[77-82]}\end{array}$ & $\begin{array}{l}\text { RCT } \\
\text { Unblinded } \\
\text { Multicentre } \\
\text { (20 centres) }\end{array}$ & 62 & $\begin{array}{l}\text { Until wound } \\
\text { healing } \\
\text { (maximum } 12 \\
\text { weeks) }\end{array}$ & 12 weeks & $\begin{array}{l}\text { Inpatient, at } \\
\text { home, } \\
\text { medical } \\
\text { practice } \\
\text { and/or } \\
\text { others }\end{array}$ & $\begin{array}{l}\text { Canada and } \\
\text { UKMarch } \\
\text { 2012-October } \\
2014\end{array}$ & $\begin{array}{l}\text { wound closure } \\
\text { Adverse events } \\
\text { pain } \\
\text { Hospital stay } \\
\text { and } \\
\text { readmission }\end{array}$ & $\begin{array}{l}\text { Subacute or chronic wounds } \\
\text { (diabetic foot ulcer, pressure } \\
\text { ulcer, venous leg ulcer or other } \\
\text { chronic) suitable for treatment } \\
\text { with NPWT }\end{array}$ \\
\hline $\begin{array}{l}\text { Chiang et al. } \\
(2017)[83]\end{array}$ & $\begin{array}{l}\text { RCT } \\
\text { Unblinded } \\
\text { Single } \\
\text { centre }\end{array}$ & 36 & Not specified & 12 months & Inpatient & $\begin{array}{l}\text { New } \\
\text { ZealandMarch } \\
\text { 2010-June } \\
2011\end{array}$ & Adverse events & $\begin{array}{l}\text { Acute open wounds } \\
\text { Patients with high risk vascular } \\
\text { foot wounds }\end{array}$ \\
\hline $\begin{array}{l}\text { Correa et al. } \\
(2016)[84,85]\end{array}$ & $\begin{array}{l}\text { RCT } \\
\text { Unblinded } \\
\text { Single } \\
\text { centre }\end{array}$ & 75 & Not specified & $\begin{array}{l}\text { Until } \\
\text { discharge } \\
\text { from hospital }\end{array}$ & Inpatient & $\begin{array}{l}\text { Columbia } \\
\text { June } 2011- \\
\text { April } 2013\end{array}$ & Mortality & $\begin{array}{l}\text { Acute open wounds } \\
\text { Traumatic open abdomen and } \\
\text { open abdomen of a medical } \\
\text { cause }\end{array}$ \\
\hline $\begin{array}{l}\text { Dalla Paola } \\
\text { et al. (2010) S-II } \\
\text { [86] }\end{array}$ & $\begin{array}{l}\text { RCT } \\
\text { Blinding } \\
\text { not } \\
\text { specified } \\
\text { Single } \\
\text { centre }\end{array}$ & 130 & $\begin{array}{l}\text { Until wound } \\
\text { healing or surgical } \\
\text { wound closure }\end{array}$ & 6 months & Inpatient & $\begin{array}{l}\text { Italy } \\
\text { July 2007-July } \\
2008\end{array}$ & $\begin{array}{l}\text { Wound closure } \\
\text { Adverse events } \\
\text { amputation }\end{array}$ & $\begin{array}{l}\text { Chronic open wounds } \\
\text { Diabetic foot wounds }\end{array}$ \\
\hline $\begin{array}{l}\text { De Laat et al. } \\
(2011)[87,88]\end{array}$ & $\begin{array}{l}\text { RCT } \\
\text { Unblinded }\end{array}$ & 24 & Not specified & $\begin{array}{l}\text { Maximum } 6 \\
\text { weeks }\end{array}$ & Inpatient & $\begin{array}{l}\text { Netherlands } \\
\text { March 2003- }\end{array}$ & Adverse events & $\begin{array}{l}\text { Chronic open wounds } \\
\text { Difficult-to-heal surgical wounds }\end{array}$ \\
\hline
\end{tabular}


Table 3 Characteristics of included studies (Continued)

\begin{tabular}{|c|c|c|c|c|c|c|c|c|}
\hline Study & $\begin{array}{l}\text { Study } \\
\text { design }\end{array}$ & $\begin{array}{l}N \\
\text { participants } \\
\text { randomised }\end{array}$ & $\begin{array}{l}\text { Duration of } \\
\text { active treatment } \\
\text { (intervention } \\
\text { group) }\end{array}$ & $\begin{array}{l}\text { Study } \\
\text { duration } \\
\text { (including } \\
\text { length of } \\
\text { follow-up) }\end{array}$ & Setting & $\begin{array}{l}\text { Location and } \\
\text { study period }\end{array}$ & $\begin{array}{l}\text { Relevant } \\
\text { outcomes }\end{array}$ & Indication/wound type \\
\hline & $\begin{array}{l}\text { Single } \\
\text { centre }\end{array}$ & & & & & March 2005 & & $\begin{array}{l}\text { or paraplegic and tetraplegic } \\
\text { patients with pressure ulcers } \\
\text { grade IV according to the } \\
\text { European Pressure Ulcer } \\
\text { Advisory Panel grading system }\end{array}$ \\
\hline DiaFu [89-95] & $\begin{array}{l}\text { RCT } \\
\text { outcome- } \\
\text { assessor } \\
\text { blinded } \\
\text { Multicentre } \\
(40 \\
\left.\text { centres }^{b}\right)\end{array}$ & 368 & $\begin{array}{l}\text { Until wound } \\
\text { healing or surgical } \\
\text { wound closure } \\
\text { (maximum } 16 \\
\text { weeks) }\end{array}$ & 6 months & $\begin{array}{l}\text { In- and } \\
\text { outpatient }\end{array}$ & $\begin{array}{l}\text { Germany } \\
\text { December } \\
2011-\text { February } \\
2015\end{array}$ & $\begin{array}{l}\text { Mortality } \\
\text { Wound closure } \\
\text { Adverse events } \\
\text { Amputation } \\
\text { Pain }\end{array}$ & $\begin{array}{l}\text { Chronic open wounds } \\
\text { Diabetic foot lesions of stadium } \\
2 \text { to } 4 \text { according to the Wagner } \\
\text { classification }\end{array}$ \\
\hline $\begin{array}{l}\text { Gupta et al. } \\
\text { (2013) [96] }\end{array}$ & $\begin{array}{l}\text { RCT } \\
\text { blinding } \\
\text { not } \\
\text { Specified } \\
\text { single } \\
\text { centre }\end{array}$ & 30 & Not specified & Not specified & Inpatient & $\begin{array}{l}\text { India } \\
\text { Study period } \\
\text { not specified }\end{array}$ & $\begin{array}{l}\text { Wound closure } \\
\text { Adverse events } \\
\text { Hospital stay } \\
\text { and } \\
\text { readmission }\end{array}$ & $\begin{array}{l}\text { Acute open wounds } \\
\text { Open musculoskeletal injuries in } \\
\text { extremities that required } \\
\text { coverage procedures }\end{array}$ \\
\hline $\begin{array}{l}\text { Hu et al. (2009) } \\
\text { [97] }\end{array}$ & $\begin{array}{l}\text { RCT } \\
\text { blinding } \\
\text { not } \\
\text { specified } \\
\text { Single } \\
\text { centre }\end{array}$ & 67 & $\begin{array}{l}\text { Until complete } \\
\text { wound healing }\end{array}$ & $\begin{array}{l}\text { Until } \\
\text { complete } \\
\text { wound } \\
\text { healing }\end{array}$ & Inpatient & $\begin{array}{l}\text { China } \\
\text { September } \\
2005- \\
\text { November } \\
2008\end{array}$ & $\begin{array}{l}\text { Wound closure } \\
\text { Adverse events } \\
\text { Amputation }\end{array}$ & $\begin{array}{l}\text { Chronic open wounds } \\
\text { Complex or refractory type } \\
\text { lesions }\end{array}$ \\
\hline $\begin{array}{l}\text { Huang et al. } \\
\text { (2006) [98] }\end{array}$ & $\begin{array}{l}\text { RCT } \\
\text { blinding } \\
\text { not } \\
\text { specified } \\
\text { Single } \\
\text { centre }\end{array}$ & 24 & $\begin{array}{l}\text { Until wound } \\
\text { closure }\end{array}$ & $\begin{array}{l}\text { until natural } \\
\text { surgery } \\
\text { wound } \\
\text { closure }\end{array}$ & inpatient & $\begin{array}{l}\text { Taiwan } \\
2004\end{array}$ & $\begin{array}{l}\text { Mortality } \\
\text { Amputation } \\
\text { Hospital stay } \\
\text { and } \\
\text { readmission }\end{array}$ & $\begin{array}{l}\text { Acute open wounds } \\
\text { Upper or lower limb of acute } \\
\text { necrotizing fasciitis }\end{array}$ \\
\hline $\begin{array}{l}\text { Jayakumar } \\
\text { et al. (2013) } \\
\text { [99] }\end{array}$ & $\begin{array}{l}\text { RCT } \\
\text { Blinding } \\
\text { not } \\
\text { specified } \\
\text { Single } \\
\text { centre }\end{array}$ & 40 & Not specified & Not specified & Inpatient & $\begin{array}{l}\text { India } \\
\text { study period } \\
\text { not specified }\end{array}$ & $\begin{array}{l}\text { Wound closure } \\
\text { Adverse events } \\
\text { Hospital stay } \\
\text { and } \\
\text { readmission }\end{array}$ & $\begin{array}{l}\text { Acute open wounds } \\
\text { Type IIIA and Type IIIB open } \\
\text { fracture both bones of leg }\end{array}$ \\
\hline $\begin{array}{l}\text { Kakagia et al. } \\
\text { (2014) [100] }\end{array}$ & $\begin{array}{l}\text { RCT } \\
\text { Blinding } \\
\text { not } \\
\text { specified } \\
\text { Single } \\
\text { centre }\end{array}$ & $\begin{array}{l}50 \text { (82 } \\
\text { wounds) }\end{array}$ & Not specified & $\begin{array}{l}\text { Average } 21 \\
\text { months } \\
\text { (range 5-36 } \\
\text { months) }\end{array}$ & Inpatient & $\begin{array}{l}\text { Greece } \\
\text { June 2006- } \\
\text { May } 2011\end{array}$ & $\begin{array}{l}\text { Wound closure } \\
\text { Adverse events }\end{array}$ & $\begin{array}{l}\text { Acute open wounds } \\
\text { Leg fasciotomies due to } \\
\text { fractures and/or soft tissue } \\
\text { injuries }\end{array}$ \\
\hline $\begin{array}{l}\text { Karatepe et al. } \\
(2011) \text { [101] }\end{array}$ & $\begin{array}{l}\text { RCT } \\
\text { Blinding } \\
\text { not } \\
\text { specified } \\
\text { Single } \\
\text { centre }\end{array}$ & 67 & Not specified & $\begin{array}{l}\text { Mean } 4 \\
\text { months } \\
\text { (range 2-8 } \\
\text { months) }\end{array}$ & Inpatient & $\begin{array}{l}\text { Turkey } \\
\text { May 2007- } \\
\text { December } \\
2008\end{array}$ & Wound closure & $\begin{array}{l}\text { Chronic open wounds } \\
\text { Biabetic foot ulcers }\end{array}$ \\
\hline $\begin{array}{l}\text { Leclercq et al. } \\
(2016) \text { [102] }\end{array}$ & $\begin{array}{l}\text { RCT } \\
\text { Unblinded } \\
\text { Single } \\
\text { centre }\end{array}$ & 46 & 5 days & 3 months & Inpatient & $\begin{array}{l}\text { France } \\
\text { October } \\
\text { 2010-May } \\
2014\end{array}$ & Wound closure & $\begin{array}{l}\text { Surgically covered wounds } \\
\text { Autologous grafting on chronic } \\
\text { leg ulcers }\end{array}$ \\
\hline $\begin{array}{l}\text { Liao et al. } \\
\text { (2012) [103] }\end{array}$ & $\begin{array}{l}\text { RCT } \\
\text { Blinding } \\
\text { not } \\
\text { specified } \\
\text { Single } \\
\text { centre }\end{array}$ & 60 & $7-10$ days & $\begin{array}{l}\text { Average } 24 \\
\text { months } \\
\text { (range 12-36 } \\
\text { months) }\end{array}$ & Inpatient & $\begin{array}{l}\text { China } \\
\text { March 2005- } \\
\text { June } 2010\end{array}$ & $\begin{array}{l}\text { Adverse events } \\
\text { Hospital stay } \\
\text { and } \\
\text { readmission }\end{array}$ & $\begin{array}{l}\text { Acute open wounds } \\
\text { Amputation wounds for limbs } \\
\text { open fractures }\end{array}$ \\
\hline $\begin{array}{l}\text { Llanos et al. } \\
\text { (2006) [104] }\end{array}$ & $\begin{array}{l}\text { RCT } \\
\text { Outcome- } \\
\text { assessor } \\
\text { blinded } \\
\text { Single } \\
\text { centre }\end{array}$ & 60 & 4 days & $7-23$ days & Inpatient & $\begin{array}{l}\text { Chile } \\
\text { May 2003- } \\
\text { October } 2004\end{array}$ & $\begin{array}{l}\text { Wound closure } \\
\text { Adverse events } \\
\text { Hospital stay } \\
\text { and } \\
\text { readmission }\end{array}$ & $\begin{array}{l}\text { Acute open wounds } \\
\text { Acute traumatic injuries and skin } \\
\text { loss which hindered primary } \\
\text { closure }\end{array}$ \\
\hline
\end{tabular}


Table 3 Characteristics of included studies (Continued)

\begin{tabular}{|c|c|c|c|c|c|c|c|c|}
\hline Study & $\begin{array}{l}\text { Study } \\
\text { design }\end{array}$ & $\begin{array}{l}N \\
\text { participants } \\
\text { randomised }\end{array}$ & $\begin{array}{l}\text { Duration of } \\
\text { active treatment } \\
\text { (intervention } \\
\text { group) }\end{array}$ & $\begin{array}{l}\text { Study } \\
\text { duration } \\
\text { (including } \\
\text { length of } \\
\text { follow-up) }\end{array}$ & Setting & $\begin{array}{l}\text { Location and } \\
\text { study period }\end{array}$ & $\begin{array}{l}\text { Relevant } \\
\text { outcomes }\end{array}$ & Indication/wound type \\
\hline $\begin{array}{l}\text { Mody et al. } \\
(2008) \text { [105] }\end{array}$ & $\begin{array}{l}\text { RCT } \\
\text { Outcome- } \\
\text { assessor } \\
\text { blinded } \\
\text { Single } \\
\text { centre }\end{array}$ & 55 & $\begin{array}{l}\text { Until discharge } \\
\text { from hospital }\end{array}$ & $\begin{array}{l}\text { Average } 26 \\
\text { days } \pm 18 \\
\text { days } \\
\text { (intervention } \\
\text { group) } \\
\text { Average } 33 \\
\text { days } \pm 37 \\
\text { days (control } \\
\text { group) }\end{array}$ & $\begin{array}{l}\text { In- and } \\
\text { outpatient }\end{array}$ & $\begin{array}{l}\text { India } \\
\text { Study period } \\
\text { not specified }\end{array}$ & $\begin{array}{l}\text { Adverse events } \\
\text { Amputation } \\
\text { Pain }\end{array}$ & $\begin{array}{l}\text { Acute and chronic open } \\
\text { wounds } \\
\text { Acute or chronic extremity } \\
\text { sacral or abdominal wound that } \\
\text { could not be treated with } \\
\text { primary closure }\end{array}$ \\
\hline $\begin{array}{l}\text { Mohsin et al. } \\
\text { (2017) [106] }\end{array}$ & $\begin{array}{l}\text { RCT } \\
\text { Outcome- } \\
\text { assessor } \\
\text { blinded } \\
\text { Single } \\
\text { centre }\end{array}$ & 100 & 4 days & $\begin{array}{l}\text { Until } \\
\text { discharge } \\
\text { from hospital }\end{array}$ & Inpatient & $\begin{array}{l}\text { India } \\
\text { January 2013- } \\
\text { December } \\
2015\end{array}$ & Adverse events & Surgically covered wounds \\
\hline $\begin{array}{l}\text { Moisidis et al. } \\
\text { (2004) [107] }\end{array}$ & $\begin{array}{l}\text { RCT } \\
\text { Outcome } \\
\text { assessor } \\
\text { blinded } \\
\text { Single } \\
\text { centre }\end{array}$ & $\begin{array}{l}22 \text { ( } 44 \text { half } \\
\text { wounds) }\end{array}$ & 5 days & 2 weeks & Inpatient & $\begin{array}{l}\text { Australia } \\
\text { July } 2001-J u l y \\
2002\end{array}$ & $\begin{array}{l}\text { wound closure } \\
\text { Adverse events }\end{array}$ & $\begin{array}{l}\text { acute or chronic open wounds } \\
\text { Split-thickness skin graft on } \\
\text { acute, subacute or chronic } \\
\text { wounds }\end{array}$ \\
\hline $\begin{array}{l}\text { Mouës et al. } \\
(2004)[108- \\
110]\end{array}$ & $\begin{array}{l}\text { RCT } \\
\text { Blinding } \\
\text { not } \\
\text { specified } \\
\text { Single } \\
\text { centre }\end{array}$ & 54 & $\begin{array}{l}\text { Until surgery } \\
\text { wound closure }\end{array}$ & Until 1 month & Inpatient & $\begin{array}{l}\text { Netherlands } \\
\text { July 1998- } \\
\text { October } 2002\end{array}$ & $\begin{array}{l}\text { Mortality } \\
\text { Wound closure } \\
\text { Adverse events }\end{array}$ & $\begin{array}{l}\text { Acute or chronic open wounds } \\
\text { Full-thickness wounds }\end{array}$ \\
\hline $\begin{array}{l}\text { Nain et al. } \\
\text { (2011) [111] }\end{array}$ & $\begin{array}{l}\text { RCT } \\
\text { Blinding } \\
\text { not } \\
\text { specified } \\
\text { Single } \\
\text { centre }\end{array}$ & 30 & $\begin{array}{l}\text { Until wound } \\
\text { closure (maximum } \\
56 \text { days) }\end{array}$ & $\begin{array}{l}\text { Maximum } 8 \\
\text { weeks }\end{array}$ & Inpatient & $\begin{array}{l}\text { India } \\
\text { Study period } \\
\text { not specified }\end{array}$ & Wound closure & $\begin{array}{l}\text { Chronic open woundsDiabetic } \\
\text { foot ulcers }\end{array}$ \\
\hline $\begin{array}{l}\text { Novinščak } \\
\text { et al. (2010) } \\
{[112]}\end{array}$ & $\begin{array}{l}\text { RCT } \\
3 \text { trial arms } \\
\text { Blinding } \\
\text { not } \\
\text { specified } \\
\text { Single } \\
\text { centre }\end{array}$ & $27^{c}$ & Not specified & 2 months & Inpatient & $\begin{array}{l}\text { Croatia } \\
\text { Study period } \\
\text { not specified }\end{array}$ & Wound closure & $\begin{array}{l}\text { Chronic open wounds } \\
\text { Complicated diabetic ulcer } \\
\text { (Wagner 2-5) }\end{array}$ \\
\hline $\begin{array}{l}\text { Perez et al. } \\
(2010) \text { [113] }\end{array}$ & $\begin{array}{l}\text { RCT } \\
\text { Unblinded } \\
\text { Single } \\
\text { centre }\end{array}$ & 49 & Not specified & $\begin{array}{l}\text { Until } 30 \text { days } \\
\text { after wound } \\
\text { healing }\end{array}$ & Inpatient & $\begin{array}{l}\text { Haiti } \\
\text { January 2007- } \\
\text { June } 2007\end{array}$ & $\begin{array}{l}\text { Wound } \\
\text { closureAdverse } \\
\text { events }\end{array}$ & $\begin{array}{l}\text { Acute and chronic open } \\
\text { wounds } \\
\text { Fasciitis of leg or forearm, } \\
\text { Fournier gangrene, abdominal } \\
\text { wound, cervical wound, inguinal } \\
\text { hernia repair, trauma to } \\
\text { extremities, venous leg ulcer }\end{array}$ \\
\hline $\begin{array}{l}\text { Rencüzoğulları } \\
\text { et al. (2015) } \\
\text { [114] }\end{array}$ & $\begin{array}{l}\text { RCT } \\
\text { Blinding } \\
\text { not } \\
\text { specified } \\
\text { Single } \\
\text { centre }\end{array}$ & 40 & Not specified & $\begin{array}{l}\text { Not } \\
\text { specified }^{d}\end{array}$ & Inpatient & $\begin{array}{l}\text { Turkey } \\
\text { February } \\
\text { 2007- } \\
\text { September } \\
2010\end{array}$ & $\begin{array}{l}\text { mortality } \\
\text { Adverse events } \\
\text { Hospital stay } \\
\text { and } \\
\text { readmission }\end{array}$ & $\begin{array}{l}\text { Acute open wounds } \\
\text { Open abdomen/decompressive } \\
\text { laparotomy as part of the } \\
\text { management of abdominal } \\
\text { compartment syndrome }\end{array}$ \\
\hline $\begin{array}{l}\text { Saaiq et al. } \\
(2010) \text { [115] }\end{array}$ & $\begin{array}{l}\text { RCT } \\
\text { Patients } \\
\text { blinded } \\
\text { Single } \\
\text { centre }\end{array}$ & 100 & 10 days & $\begin{array}{l}\text { Until wound } \\
\text { healing }\end{array}$ & Inpatient & $\begin{array}{l}\text { Pakistan } \\
\text { October } \\
\text { 2007- } \\
\text { December } \\
2009\end{array}$ & $\begin{array}{l}\text { Mortality } \\
\text { Wound closure } \\
\text { Adverse events } \\
\text { Hospital stay } \\
\text { and } \\
\text { readmission }\end{array}$ & $\begin{array}{l}\text { Acute open wounds } \\
\text { Acute traumatic wounds most } \\
\text { frequently located on the lower } \\
\text { limb, upper limb, trunk and } \\
\text { scalp }\end{array}$ \\
\hline $\begin{array}{l}\text { Shen et al. } \\
\text { (2013) [116] }\end{array}$ & $\begin{array}{l}\text { RCT } \\
\text { Blinding } \\
\text { not } \\
\text { specified } \\
\text { Single } \\
\text { centre }\end{array}$ & 307 & 6 days & Not specified $^{e}$ & Inpatient & $\begin{array}{l}\text { China } \\
\text { August 2009- } \\
\text { May } 2012\end{array}$ & Wound closure & $\begin{array}{l}\text { Acute open wounds } \\
\text { Superficial partial thickness scald } \\
\text { in children, shallow second } \\
\text { degree burns mainly being } \\
\text { located on the thorax, abdomen } \\
\text { and limbs }\end{array}$ \\
\hline
\end{tabular}


Table 3 Characteristics of included studies (Continued)

\begin{tabular}{|c|c|c|c|c|c|c|c|c|}
\hline Study & $\begin{array}{l}\text { Study } \\
\text { design }\end{array}$ & $\begin{array}{l}N \\
\text { participants } \\
\text { randomised }\end{array}$ & $\begin{array}{l}\text { Duration of } \\
\text { active treatment } \\
\text { (intervention } \\
\text { group) }\end{array}$ & $\begin{array}{l}\text { Study } \\
\text { duration } \\
\text { (including } \\
\text { length of } \\
\text { follow-up) }\end{array}$ & Setting & $\begin{array}{l}\text { Location and } \\
\text { study period }\end{array}$ & $\begin{array}{l}\text { Relevant } \\
\text { outcomes }\end{array}$ & Indication/wound type \\
\hline $\begin{array}{l}\text { Sibin et al. } \\
\text { (2017) [117] }\end{array}$ & $\begin{array}{l}\text { RCT } \\
\text { Blinding } \\
\text { not } \\
\text { specified } \\
\text { Single } \\
\text { centre }\end{array}$ & 30 & Not specified & Not specified & Inpatient & $\begin{array}{l}\text { IndiaJanuary } \\
\text { 2015-July } \\
2015\end{array}$ & $\begin{array}{l}\text { Wound closure } \\
\text { Adverse events } \\
\text { Hospital stay } \\
\text { and } \\
\text { readmission }\end{array}$ & $\begin{array}{l}\text { Scute open wounds } \\
\text { Gustilo type IIIA or IIIB open } \\
\text { tibia fractures }\end{array}$ \\
\hline $\begin{array}{l}\text { Sinha et al. } \\
\text { (2013) [118] }\end{array}$ & $\begin{array}{l}\text { RCT } \\
\text { Outcome } \\
\text { assessor } \\
\text { blinded } \\
\text { Single } \\
\text { centre }\end{array}$ & 30 & Not specified & Not specified ${ }^{f}$ & Inpatient & $\begin{array}{l}\text { India } \\
2011-2012\end{array}$ & Adverse events & $\begin{array}{l}\text { Acute open wounds } \\
\text { Open musculoskeletal injuries in } \\
\text { extremities according to Gustilo } \\
\text { Anderson classification grade II, } \\
\text { IIIA, IIIB and IIIC }\end{array}$ \\
\hline $\begin{array}{l}\text { SWHSI [119- } \\
121]\end{array}$ & $\begin{array}{l}\text { RCT } \\
\text { Outcome } \\
\text { assessor } \\
\text { blinded } \\
\text { Multicentre } \\
\text { (3 centres) }\end{array}$ & 40 & Not specified & 3 months & $\begin{array}{l}\text { In- and } \\
\text { Outpatient }\end{array}$ & $\begin{array}{l}\text { UK } \\
\text { November } \\
\text { 2015- } \\
\text { September } \\
2016\end{array}$ & $\begin{array}{l}\text { Wound closure } \\
\text { Adverse events } \\
\text { Amputation } \\
\text { Pain } \\
\text { Hospital stay } \\
\text { and } \\
\text { readmission } \\
\text { Health-related } \\
\text { quality of life }\end{array}$ & $\begin{array}{l}\text { Acute open wounds } \\
\text { Surgical wounds on the foot, } \\
\text { abdomen, leg, breast, groyne, } \\
\text { buttocks or perianal area }\end{array}$ \\
\hline $\begin{array}{l}\text { TOPSKIN [122- } \\
124]\end{array}$ & $\begin{array}{l}\text { RCT } \\
4 \text { trial Arms } \\
\text { Unblinded } \\
\text { Multicentre } \\
\text { (3 centres) }\end{array}$ & 86 & Not specified & 12 months & Inpatient & $\begin{array}{l}\text { Netherlands } \\
\text { October } \\
\text { 2007-February } \\
2010\end{array}$ & $\begin{array}{l}\text { Adverse events } \\
\text { Pain } \\
\text { Hospital stay } \\
\text { and } \\
\text { readmission }\end{array}$ & $\begin{array}{l}\text { Acute open wounds } \\
\text { Deep dermal or full-thickness } \\
\text { burns of arm, leg or trunk re- } \\
\text { quiring skin transplantation }\end{array}$ \\
\hline $\begin{array}{l}\text { VAC (2001-01) } \\
{[125-127]}\end{array}$ & $\begin{array}{l}\text { RCT } \\
\text { Outcome- } \\
\text { assessor } \\
\text { blinded } \\
\text { Multicentre } \\
\text { (25 centres) }\end{array}$ & $263^{9}$ & $\begin{array}{l}\text { Until surgery } \\
\text { wound closure or } \\
\text { wound healing } \\
\text { with secondary } \\
\text { intention } \\
\text { (maximum } 84 \\
\text { days) }\end{array}$ & $\begin{array}{l}\text { Maximum } 12 \\
\text { months }\end{array}$ & $\begin{array}{l}\text { In- and } \\
\text { outpatient }\end{array}$ & $\begin{array}{l}\text { Canada and } \\
\text { USA } \\
\text { August 2001- } \\
\text { October } 2006\end{array}$ & $\begin{array}{l}\text { Mortality } \\
\text { Adverse events }\end{array}$ & $\begin{array}{l}\text { Chronic open wounds } \\
\text { Stage III and IV pressure ulcers } \\
\text { according to the National } \\
\text { Pressure Advisory Panel (NPUAP) } \\
\text { staging system located on the } \\
\text { trunk or trochanter region }\end{array}$ \\
\hline $\begin{array}{l}\operatorname{VAC}(2001-02) \\
{[126,128,129]}\end{array}$ & $\begin{array}{l}\text { RCT } \\
\text { Outcome- } \\
\text { assessor } \\
\text { blinded } \\
\text { Multicentre } \\
\text { (29 centres) }\end{array}$ & 208 & $\begin{array}{l}\text { Until wound } \\
\text { healing with } \\
\text { secondary } \\
\text { intention } \\
\text { (maximum } 112 \\
\text { days) }\end{array}$ & $\begin{array}{l}\text { Maximum } 12 \\
\text { months }\end{array}$ & Inpatient ${ }^{\text {h }}$ & $\begin{array}{l}\text { USA } \\
\text { January 2002- } \\
\text { July } 2005\end{array}$ & Adverse events & $\begin{array}{l}\text { Chronic open wounds } \\
\text { Venous stasis ulcers }\end{array}$ \\
\hline $\begin{array}{l}\text { VAC }(2001-03) \\
{[130,131]}\end{array}$ & $\begin{array}{l}\text { RCT } \\
\text { Outcome- } \\
\text { assessor } \\
\text { blinded } \\
\text { Multicentre }\end{array}$ & 12 & Not specified & 90 days & $\begin{array}{l}\text { Not } \\
\text { specified }\end{array}$ & $\begin{array}{l}\text { USA } \\
\text { October } \\
2001-J u l y \\
2004\end{array}$ & $\begin{array}{l}\text { Wound closure } \\
\text { Adverse events }\end{array}$ & $\begin{array}{l}\text { Shronic open wounds } \\
\text { Split thickness skin graft closure } \\
\text { of venous stasis ulcers }\end{array}$ \\
\hline $\begin{array}{l}\text { VAC (2001-06) } \\
{[132-134]}\end{array}$ & $\begin{array}{l}\text { RCT } \\
\text { Unblinded } \\
\text { Single } \\
\text { centre }\end{array}$ & $\begin{array}{l}58 \text { (62 } \\
\text { wounds) }\end{array}$ & $\begin{array}{l}\text { Until surgery } \\
\text { wound closure }\end{array}$ & $\begin{array}{l}\text { Average } 28 \\
\text { months } \\
\text { (range 14-67 } \\
\text { months) }\end{array}$ & Inpatient & $\begin{array}{l}\text { USA } \\
\text { June 2001- } \\
\text { August } 2006\end{array}$ & $\begin{array}{l}\text { Wound closure } \\
\text { Adverse events } \\
\text { Amputation } \\
\text { Hospital stay } \\
\text { and } \\
\text { readmission }\end{array}$ & $\begin{array}{l}\text { Acute open wounds } \\
\text { Severe open fractures including } \\
\text { type II fractures, type IIIA } \\
\text { fractures that were either } \\
\text { heavily contaminated or had a } \\
\text { remarkably severe soft tissue } \\
\text { injury, and all type IIIB or IIIC } \\
\text { fractures according to the } \\
\text { classification of Gustilo and } \\
\text { Anderson }\end{array}$ \\
\hline $\begin{array}{l}\text { VAC }(2001-07) \\
{[135-141]}\end{array}$ & $\begin{array}{l}\mathrm{RCT} \\
\text { Outcome } \\
\text { assessor } \\
\text { blinded } \\
\text { Multicentre } \\
\text { (19 centres) }\end{array}$ & $164^{i}$ & $\begin{array}{l}\text { Until wound } \\
\text { closure (maximum } \\
112 \text { days) }\end{array}$ & $\begin{array}{l}\text { Maximum } 13 \\
\text { months }^{j}\end{array}$ & Inpatient ${ }^{\text {h }}$ & $\begin{array}{l}\text { USA } \\
\text { August 2002- } \\
\text { November } \\
2005\end{array}$ & $\begin{array}{l}\text { Mortality } \\
\text { Wound closure } \\
\text { Adverse events } \\
\text { Hospital stay } \\
\text { and } \\
\text { readmission }\end{array}$ & $\begin{array}{l}\text { Chronic open wounds } \\
\text { Diabetic foot amputation } \\
\text { wound up to the } \\
\text { transmetatarsal region of the } \\
\text { foot }\end{array}$ \\
\hline $\begin{array}{l}\text { VAC (2001-08) } \\
{[142-149]}\end{array}$ & $\begin{array}{l}\text { RCT } \\
\text { Unblinded } \\
\text { Multicentre } \\
\text { (29 centres) }\end{array}$ & 335 & $\begin{array}{l}\text { Until wound } \\
\text { closure (maximum } \\
112 \text { days) }\end{array}$ & $\begin{array}{l}\text { Maximum } 12 \\
\text { months }\end{array}$ & Inpatient ${ }^{\text {h }}$ & $\begin{array}{l}\text { Canada and } \\
\text { USA } \\
\text { August 2002- } \\
\text { August 2005 }\end{array}$ & $\begin{array}{l}\text { Mortality } \\
\text { Wound closure } \\
\text { Adverse events }\end{array}$ & $\begin{array}{l}\text { Chronic open wounds } \\
\text { Diabetic foot ulcer equivalent to } \\
\text { Stage } 2 \text { or greater as defined by } \\
\text { Wagner's Scale }\end{array}$ \\
\hline VAC (2002-09) & $\mathrm{RCT}$ & 54 & Until surgery & Maximum 6 & Inpatient ${ }^{\text {h }}$ & Canada and & Mortality & Acute open wounds \\
\hline
\end{tabular}


Table 3 Characteristics of included studies (Continued)

\begin{tabular}{|c|c|c|c|c|c|c|c|c|}
\hline Study & $\begin{array}{l}\text { Study } \\
\text { design }\end{array}$ & $\begin{array}{l}N \\
\text { participants } \\
\text { randomised }\end{array}$ & $\begin{array}{l}\text { Duration of } \\
\text { active treatment } \\
\text { (intervention } \\
\text { group) }\end{array}$ & $\begin{array}{l}\text { Study } \\
\text { duration } \\
\text { (including } \\
\text { length of } \\
\text { follow-up) }\end{array}$ & Setting & $\begin{array}{l}\text { Location and } \\
\text { study period }\end{array}$ & $\begin{array}{l}\text { Relevant } \\
\text { outcomes }\end{array}$ & Indication/wound type \\
\hline$[126,150,151]$ & $\begin{array}{l}\text { Outcome } \\
\text { assessor } \\
\text { blinded } \\
\text { Multicentre } \\
\text { (14 centres) }\end{array}$ & & $\begin{array}{l}\text { wound closure or } \\
\text { wound healing } \\
\text { with secondary } \\
\text { intention } \\
\text { (maximum } 84 \\
\text { days) }\end{array}$ & months & & $\begin{array}{l}\text { USA } \\
\text { October } \\
\text { 2002-July } \\
2005\end{array}$ & $\begin{array}{l}\text { Wound closure } \\
\text { Adverse events }\end{array}$ & Open chest wounds \\
\hline $\begin{array}{l}\text { VAC }(2002-10) \\
{[126,152,153]}\end{array}$ & $\begin{array}{l}\text { RCT } \\
\text { Outcome } \\
\text { assessor } \\
\text { blinded } \\
\text { Multicentre } \\
\text { (19 centres) }\end{array}$ & 134 & $\begin{array}{l}\text { Until surgery } \\
\text { wound closure or } \\
\text { wound healing } \\
\text { with secondary } \\
\text { intention } \\
\text { (maximum } 84 \\
\text { days) }\end{array}$ & $\begin{array}{l}\text { Maximum } 6 \\
\text { months }\end{array}$ & Inpatient ${ }^{\mathrm{h}}$ & $\begin{array}{l}\text { Canada, } \\
\text { Mexico and } \\
\text { USA } \\
\text { June 2002-- } \\
\text { October 2004 }\end{array}$ & $\begin{array}{l}\text { Mortality } \\
\text { Wound closure } \\
\text { Adverse events }\end{array}$ & $\begin{array}{l}\text { Acute open wounds } \\
\text { Open abdominal wounds }\end{array}$ \\
\hline $\begin{array}{l}\text { Virani et al. } \\
\text { (2016) [154] }\end{array}$ & $\begin{array}{l}\text { RCT } \\
\text { Blinding } \\
\text { not } \\
\text { specified } \\
\text { Single } \\
\text { centre }\end{array}$ & 93 & $\begin{array}{l}\text { Until sufficient } \\
\text { granulation tissue } \\
\text { is present or } \\
\text { approximation of } \\
\text { the wound } \\
\text { margins }\end{array}$ & $\begin{array}{l}\text { Average } 23 \\
\text { weeks } \pm 6 \\
\text { weeks }\end{array}$ & Inpatient & $\begin{array}{l}\text { India } \\
\text { Study period } \\
\text { not specified }\end{array}$ & $\begin{array}{l}\text { Wound closure } \\
\text { Adverse events }\end{array}$ & $\begin{array}{l}\text { Acute open wounds } \\
\text { Open diaphyseal tibial fractures, } \\
\text { the majority of which were } \\
\text { Gustilo Anderson Grade II and } \\
\text { Grade IIIA fractures with heavy } \\
\text { contamination and severe soft } \\
\text { tissue and bony injury along } \\
\text { with all Grade IIIB and Grade IIIC } \\
\text { fractures }\end{array}$ \\
\hline $\begin{array}{l}\text { Vuerstaek et al. } \\
\text { (2006) [155- } \\
157]\end{array}$ & $\begin{array}{l}\text { RCT } \\
\text { Blinding } \\
\text { not } \\
\text { specified } \\
\text { Multicentre } \\
\text { (2 centres) }\end{array}$ & 60 & Maximum 4 days & 12 months & Inpatient & $\begin{array}{l}\text { Netherlands } \\
\text { May } 2001- \\
\text { May } 2003\end{array}$ & $\begin{array}{l}\text { Mortality } \\
\text { Wound closure } \\
\text { Adverse events } \\
\text { Pain } \\
\text { Hospital stay } \\
\text { and } \\
\text { readmission }\end{array}$ & $\begin{array}{l}\text { Chronic open wounds } \\
\text { Chronic venous, combined } \\
\text { venous and arterial, or } \\
\text { microangiopathic } \\
\text { (arteriolosclerotic) leg ulcers of } \\
>6 \text { months' duration }\end{array}$ \\
\hline $\begin{array}{l}\text { WOLLF [158- } \\
\text { 161] }\end{array}$ & $\begin{array}{l}\text { RCT } \\
\text { Outcome } \\
\text { assessor } \\
\text { blinded } \\
\text { Multicentre } \\
\text { (24 centres) }\end{array}$ & $460^{k}$ & $\begin{array}{l}\text { Until wound } \\
\text { closure or surgical } \\
\text { covering }\end{array}$ & 12 months & Inpatient & $\begin{array}{l}\text { UK } \\
\text { 07/2012- } \\
\text { 2012/2015 }\end{array}$ & $\begin{array}{l}\text { Mortality } \\
\text { Wound closure } \\
\text { Adverse events } \\
\text { Amputation } \\
\text { Pain } \\
\text { Health-related } \\
\text { quality of life } \\
\text { Physical } \\
\text { function }\end{array}$ & $\begin{array}{l}\text { Acute open wounds } \\
\text { Severe open fracture of the } \\
\text { lower limb. Wounds were } \\
\text { graded as a Gustilo and } \\
\text { Anderson II or III }\end{array}$ \\
\hline $\begin{array}{l}\text { Xu et al. (2015) } \\
{[162]}\end{array}$ & $\begin{array}{l}\text { RCT } \\
\text { Blinding } \\
\text { not } \\
\text { specified } \\
\text { Single } \\
\text { centre }\end{array}$ & 40 & $3-5$ days & Not specified' & Inpatient & $\begin{array}{l}\text { China } \\
\text { 09/2013- } \\
\text { 2009/2014 }\end{array}$ & $\begin{array}{l}\text { Mortality } \\
\text { Wound closure } \\
\text { Adverse events } \\
\text { Hospital stay } \\
\text { and } \\
\text { readmission }\end{array}$ & $\begin{array}{l}\text { Acute open wounds } \\
\text { Necrotizing fasciitis in the } \\
\text { inguinal region or genital area }\end{array}$ \\
\hline
\end{tabular}

Study title in italics : study unpublished

$R C T$ randomised controlled trial

${ }^{a}$ Data from www.ClinicalTrials.gov, 5 to 20 study centres are listed in the study protocol

${ }^{\mathrm{b}}$ Number of study centres where patients were enrolled

'IQWiG's own calculation

'The authors' presentation indicates that the patients were observed until they were discharged from hospital. Accordingly, the intervention group was observed for an average of 28.5 days \pm 21.3 days and the control group for an average of 27.4 days \pm 25.3 days

'The authors' presentation indicates that the patients were observed until they were discharged from hospital. However, no further details can be found. Only the data on time to wound healing with an average of 9.2 days \pm 0.6 days in the intervention group and an average of 10.1 days \pm 1.6 days in the control group allow an approximate estimation of the study duration

'The authors' presentation indicates that the patients were observed for 8 days

${ }^{9}$ Seven patients received no intervention

${ }^{\mathrm{h}}$ The information provided indicates that at least outpatient aftercare was provided as part of the study. There are no explicit statements on the outpatient use of NPWT

'Two patients received no intervention

${ }^{\mathrm{j}}$ For patients with wound healing. Patients without wound healing were not monitored after the maximum treatment duration of 112 days

kOriginally, 625 patients were randomised, but due to the severity of the disease, only 460 patients were included in the study

'The authors' presentation indicates that the patients were observed until they were discharged from hospital. Accordingly, the intervention group was observed for an average of 21 days \pm 1.9 days and the control group for an average of 32 days \pm 2.8 days 
Table 4 Risk of bias of included studies

\begin{tabular}{|c|c|c|c|c|c|c|c|c|}
\hline \multirow[t]{2}{*}{ Study } & \multirow{2}{*}{$\begin{array}{l}\text { Randomisation } \\
\text { appropriate }\end{array}$} & \multirow{2}{*}{$\begin{array}{l}\text { Allocation } \\
\text { concealment } \\
\text { appropriate }\end{array}$} & \multicolumn{2}{|l|}{ Blinding } & \multirow{2}{*}{$\begin{array}{l}\text { Selective } \\
\text { reporting } \\
\text { improbable }\end{array}$} & \multirow{2}{*}{$\begin{array}{l}\text { Absence of } \\
\text { other } \\
\text { factors } \\
\text { potentially } \\
\text { causing } \\
\text { bias }\end{array}$} & \multirow{2}{*}{$\begin{array}{l}\text { Risk } \\
\text { of } \\
\text { bias: } \\
\text { study } \\
\text { level } \\
\text { a }\end{array}$} & \multirow{2}{*}{$\begin{array}{l}\text { Risk of } \\
\text { bias: } \\
\text { outcome }_{\text {level }^{\text {b }}}\end{array}$} \\
\hline & & & Patients & Treating staff & & & & \\
\hline Acosta et al. (2013) [65-67] & Yes & Unclear & n. a. & n. a. & n. a. & n. a. & High & High \\
\hline Arti et al. (2016) [68,69] & Unclear & Unclear & n. a. & n. a. & n. a. & n. a. & High & High \\
\hline Ashby et al. (2012) [70, 71] & Yes & Yes & no & no & unclear & yes & Low & Low/high ${ }^{c}$ \\
\hline Banasiewicz et al. (2013) [72] & Unclear & Unclear & n. a. & n. a. & n. a. & n. a. & High & High \\
\hline Bee et al. (2008) [73] & Unclear & Unclear & n. a. & n. a. & n. a. & n. a. & High & High \\
\hline Biter et al. (2014) [74, 75] & Yes & Unclear & n. a. & n. a. & n. a. & n. a. & High & High \\
\hline Braakenburg et al. (2006) [76] & Yes & Unclear & n. a. & n. a. & n. a. & n. a. & High & High \\
\hline CE/044/PIC [77-82] & Unclear & Unclear & n. a. & n. a. & n. a. & n. a. & High & High \\
\hline Chiang et al. (2017) [83] & Yes & Unclear & n. a. & n. a. & n. a. & n. a. & High & High \\
\hline Correa et al. (2016) [84, 85] & Yes & Unclear & n. a. & n. a. & n. a. & n. a. & High & High \\
\hline Dalla Paola et al. (2010) S-II [86] & Yes & Unclear & n. a. & n. a. & n. a. & n. a. & High & High \\
\hline De Laat et al. (2011) [87, 88] & Unclear & Unclear & n. a. & n. a. & n. a. & n. a. & High & High \\
\hline DiaFu [89-95] & Yes & Yes & no & no & yes & yes & Low & High \\
\hline Gupta et al. (2013) [96] & Unclear & Unclear & n. a. & n. a. & n. a. & n. a. & High & High \\
\hline Hu et al. (2009) [97] & Yes & Unclear & n. a. & n. a. & n. a. & n. a. & High & High \\
\hline Huang et al. (2006) [98] & Unclear & Unclear & n. a. & n. a. & n. a. & n. a. & High & High \\
\hline Jayakumar et al. (2013) [99] & Unclear & Unclear & n. a. & n. a. & n. a. & n. a. & High & High \\
\hline Kakagia et al. (2014) [100] & Yes & Unclear & n. a. & n. a. & n. a. & n. a. & High & High \\
\hline Karatepe et al. (2011) [101] & Yes & Unclear & n. a. & n. a. & n. a. & n. a. & High & High \\
\hline Leclercq et al. (2016) [102] & Unclear & Unclear & n. a. & n. a. & n. a. & n. a. & High & High \\
\hline Liao et al. (2012) [103] & Yes & Unclear & n. a. & n. a. & n. a. & n. a. & High & High \\
\hline Llanos et al. (2006) [104] & Yes & Yes & no & no & unclear & yes & Low & Low \\
\hline Mody et al. (2008) [105] & Yes & Unclear & n. a. & n. a. & n. a. & n. a. & High & High \\
\hline Mohsin et al. (2017) [106] & Yes & Unclear & n. a. & n. a. & n. a. & n. a. & High & High \\
\hline Moisidis et al. (2004) [107] & Unclear & Unclear & n. a. & n. a. & n. a. & n. a. & High & High \\
\hline Mouës et al. (2004) [108-110] & Unclear & Unclear & n. a. & n. a. & n. a. & n. a. & High & High \\
\hline Nain et al. (2011) [111] & Unclear & Unclear & n. a. & n. a. & n. a. & n. a. & High & High \\
\hline Novinščak et al. (2010) [112] & Unclear & Unclear & n. a. & n. a. & n. a. & n. a. & High & High \\
\hline Perez et al. (2010) [113] & Unclear & Unclear & n. a. & n. a. & n. a. & n. a. & High & High \\
\hline Rencüzoğulları et al. (2015) [114] & Unclear & Unclear & n. a. & n. a. & n. a. & n. a. & High & High \\
\hline Saaiq et al. (2010) [115] & Yes & Unclear & n. a. & n. a. & n. a. & n. a. & High & High \\
\hline Shen et al. (2013) [116] & Yes & Unclear & n. a. & n. a. & n. a. & n. a. & High & High \\
\hline Sibin et al. (2017) [117] & Unclear & Unclear & n. a. & n. a. & n. a. & n. a. & High & High \\
\hline Sinha et al. (2013) [118] & Unclear & Unclear & n. a. & n. a. & n. a. & n. a. & High & High \\
\hline SWHSI [119-121] & Yes & Yes & no & no & yes & yes & Low & Low/high $^{d}$ \\
\hline TOPSKIN [122-124] & Unclear & Yes & no & no & no & yes & High & High \\
\hline VAC (2001-01) [125-127] & Yes & Yes & no & no & no & no ${ }^{e}$ & High & High \\
\hline VAC (2001-02) [126, 128, 129] & Yes & Yes & no & no & no & $n o^{f}$ & High & High \\
\hline $\operatorname{VAC}(2001-03)[130,131]$ & Unclear & Unclear & n. a. & n. a. & n. a. & n. a. & High & High \\
\hline VAC (2001-06) [132-134] & Yes & Unclear & n. a. & n. a. & n. a. & n. a. & High & High \\
\hline VAC (2001-07) [135-141] & Yes & Yes & No & No & Yes & Yes & Low & High \\
\hline
\end{tabular}


Table 4 Risk of bias of included studies (Continued)

\begin{tabular}{|c|c|c|c|c|c|c|c|c|}
\hline \multirow[t]{2}{*}{ Study } & \multirow{2}{*}{$\begin{array}{l}\text { Randomisation } \\
\text { appropriate }\end{array}$} & \multirow{2}{*}{$\begin{array}{l}\text { Allocation } \\
\text { concealment } \\
\text { appropriate }\end{array}$} & \multicolumn{2}{|l|}{ Blinding } & \multirow{2}{*}{$\begin{array}{l}\text { Selective } \\
\text { reporting } \\
\text { improbable }\end{array}$} & \multirow{2}{*}{$\begin{array}{l}\text { Absence of } \\
\text { other } \\
\text { factors } \\
\text { potentially } \\
\text { causing } \\
\text { bias }\end{array}$} & \multirow{2}{*}{$\begin{array}{l}\text { Risk } \\
\text { of } \\
\text { bias: } \\
\text { study } \\
\text { level } \\
\text { a }\end{array}$} & \multirow{2}{*}{$\begin{array}{l}\text { Risk of } \\
\text { bias: } \\
\text { outcome } \\
\text { level }^{\mathrm{b}}\end{array}$} \\
\hline & & & Patients & Treating staff & & & & \\
\hline VAC (2001-08) [142-149] & Yes & Yes & No & No & No & Yes & High & High \\
\hline VAC (2002-09) [126, 150, 151] & Yes & Unclear & n. a. & n. a. & n. a. & n. a. & High & High \\
\hline VAC (2002-10) [126, 152, 153] & Yes & Unclear & n. a. & n. a. & n. a. & n. a. & High & High \\
\hline Virani et al. (2016) [154] & Yes & Unclear & n. a. & n. a. & n. a. & n. a. & High & High \\
\hline Vuerstaek et al. (2006) [155-157] & Yes & Yes & No & No & Yes & Yes & Low & High \\
\hline WOLLF [158-161] & Yes & Yes & No & No & Unclear & Yes & Low & High \\
\hline Xu et al. (2015) [162] & Unclear & Unclear & n. a. & n. a. & n. a. & n. a. & High & High \\
\hline
\end{tabular}

n. a. not applied

af the evaluation of the items "random sequence generation" and "allocation concealment" revealed a high risk of bias, no further evaluations of the remaining items were performed

${ }^{\mathrm{b}}$ Details are given in Tables 1 to 8 in Additional file 2

'The outcomes pain and adverse events (wound bleeding and infection) showed a high risk of bias

${ }^{\mathrm{d}}$ The outcomes adverse events, pain, duration of hospital stay and quality of life showed a high risk of bias

${ }^{\mathrm{e}}$ In the control group and in the NPWT group, $27.8 \%$ and $7.7 \%$ of the patients respectively discontinued the study due to treatment failure. The time point of study discontinuation was documented as the final study visit date

fOnly data from 146 out of 205 randomised patients available (71.2\%)

\section{Effects of NPWT versus SWT}

Wound closure was measured as wound healing (yes/no), time to wound healing (in days), wound healing and/or surgical wound closure (yes/no), time to wound healing and/or surgical wound closure (in days), time to wound healing after the intervention and surgical wound closure $(<6$ weeks yes/no), and time to wound healing after the intervention and surgical wound closure (in days).

The results for wound healing (yes/no) were reported in 14 studies (Acosta 2013, Ashby 2012, Braakenburg 2006, CE/044/PIC, Dalla Paola 2010 S-II, Hu 2009, Leclercq 2016, Llanos 2006, Moisidis 2004, Novinščak 2010, VAC 2001-03, VAC 2001-07, VAC 2001-08 and Vuerstaek 2006). Two studies with a low risk of bias (Ashby 2012 and Llanos 2006) showed no statistically significant difference between the groups. The combined analysis of studies with a low and high risk of bias showed a statistically significant effect in favour of NPWT (OR 1.56, 95\% CI 1.15 to $2.13, p=0.008$, see Fig. 2).

Only 7 studies defined wound healing as $100 \%$ reepithelialization (Acosta 2013, CE/044/PIC, Dalla Paola 2010 S-II, Moisidis 2004, VAC 2001-07, VAC 2001-08 and Vuerstaek 2006), while in the other 7 , a definition was missing. We therefore conducted a sensitivity analysis with the definition of wound healing as a stratification factor. Studies with a proper definition showed a statistically significant effect in favour of NPWT (OR 1.53, 95\% CI 1.04 to $2.23, p=0.034)$. Studies without such a definition showed no statistically significant effect (OR 1.72, 95\% CI 0.73 to $4.04, p=0.163$ ). However, the interaction test showed no statistically significant difference between the two effects $(p=0.743)$. There was an indication of a greater effect of NPWT on wound healing.

Six studies reported data on time to wound healing (in days) (Acosta 2013, Biter 2014, Karatepe 2011, Llanos 2006, Shen 2013 and Vuerstaek 2006). The study with a low risk of bias (Llanos 2006) (Hedges' g - 1.33, 95\% CI -1.90 to -0.77 , see Fig. 3), as well as studies with a low and high risk of bias (Hedges' g $-0.77,95 \%$ CI -1.19 to $-0.35, p=0.005$, see Fig. 3) showed a statistically significant difference in favour of NPWT. The results were classified as clinically relevant, since the upper limits of these 95\% CIs were below the irrelevance threshold of 0.2. A sensitivity analysis stratified by the definition of wound healing showed a statistically significant result for the 4 studies (Acosta 2013, Biter 2014, Shen 2013 and Vuerstaek 2006) with a proper definition (100\% reepithelialization; Hedges' g $-0.69,95 \%$ CI -0.88 to $0.49, p<0.001)$ and the 2 studies with a missing definition (Hedges' $g-0.95,95 \% \mathrm{CI}-1.33$ to $-0.58, p<$ $0.001)$. However, the interaction test showed no statistically significant difference between the two effects $(p=$ 0.210). There was proof of a greater effect of NPWT on time to wound healing.

The meta-analysis of time to wound healing after the intervention and surgical wound closure (< 6 weeks yes) no) included 3 studies with a high risk of bias (Gupta 2013, Jayakumar 2013 and Sibin 2017). There was a statistically significant effect in favour of NPWT after 6 weeks (OR 16.07, 95\% CI 3.19 to 80.97, $p=0.018$, see Fig. 4).

The meta-analysis of time to wound healing after the intervention and surgical wound closure (in days) included 


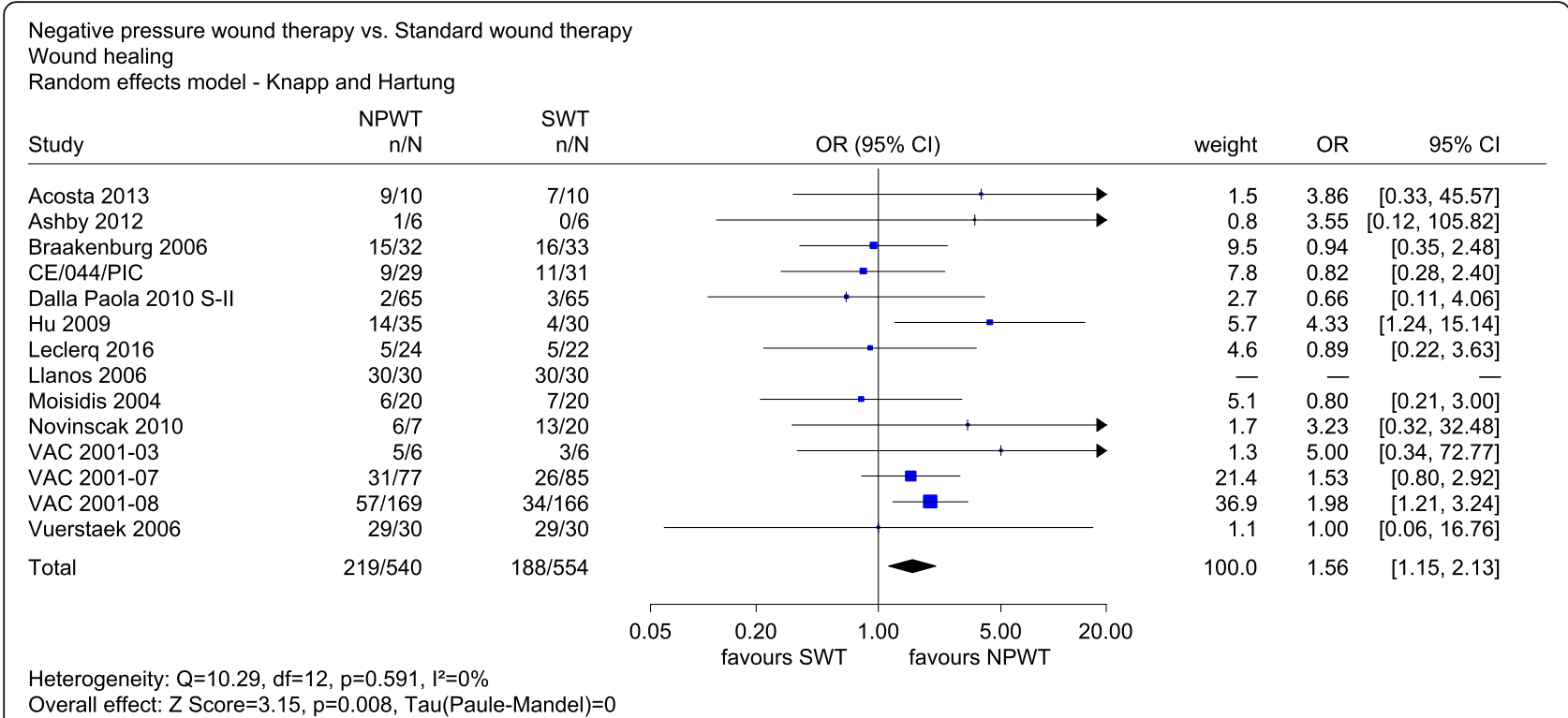

Fig. 2 Forest plot of wound healing with overall effect estimation, NPWT vs. SWT. Abbreviations: Cl confidence interval, $n$ number of events, $N$ number of patients, NPWT negative pressure wound therapy, OR odds ratio, SWT standard wound therapy

2 studies with a high risk of bias (Dalla Paola 2010 S-II and $\mathrm{Hu}$ 2009). There was a statistically significant difference in favour of NPWT (Hedges' $g-1.14,95 \% \mathrm{CI}-1.45$ to $-0.84, p<0.001$, see Fig. 5). The effect was clinically relevant, since the upper limit of the $95 \%$ CI was below the irrelevance threshold of - 0.2. Overall, there was an indication of a greater effect of NPWT on time to wound healing after the intervention and surgical closure.

Due to heterogeneity, no effect estimate was calculated for wound healing and/or surgical wound closure (yes/ no) (21 studies; Acosta 2013, Arti 2016, Braakenburg 2006, Dalla Paola 2010 S-II, DiaFu, Gupta 2013, Hu 2009, Jayakumar 2013, Kakagia 2014, Mouës 2004, Nain 2011, Perez 2010, Saaiq 2010, Sibin 2017, SWHSI, VAC 2001-06, VAC 2001-07, VAC 2001-08, Virani 2016, WOLLF and $\mathrm{Xu} 2015)$ and time to wound healing and/ or surgical wound closure (in days) (9 studies; Braakenburg 2006, Mouës 2004, Perez 2010, VAC 2001-07, VAC 2001-08, VAC 2002-09, VAC 2002-10, Virani 2016 and Vuerstaek 2006). In both analyses, the 95\% PI included

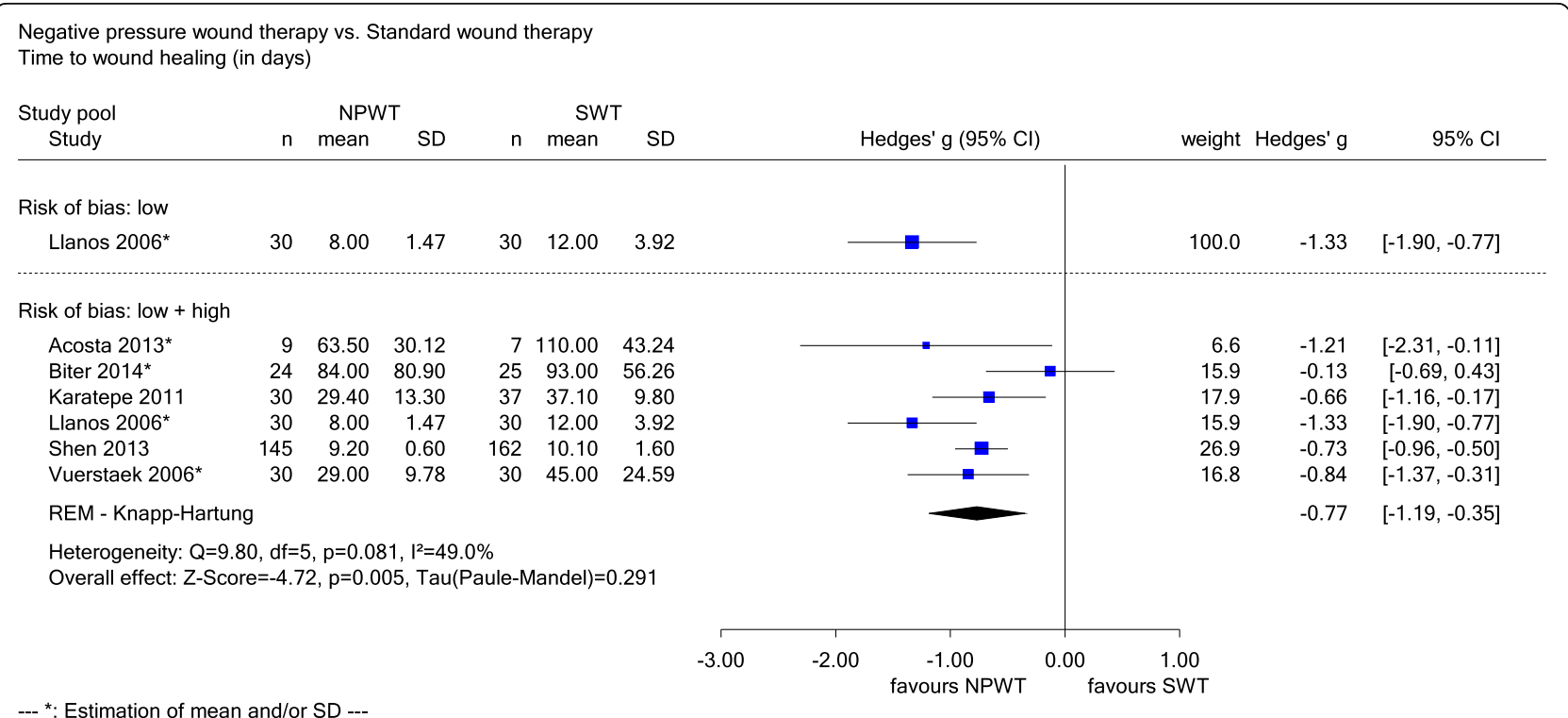

Fig. 3 Forest plot of time to wound healing (in days) with overall effect estimation, NPWT vs. SWT. Abbreviations: Cl confidence interval, $n$ number of patients, NPWT negative pressure wound therapy, SD standard deviation, SWT standard wound therapy 


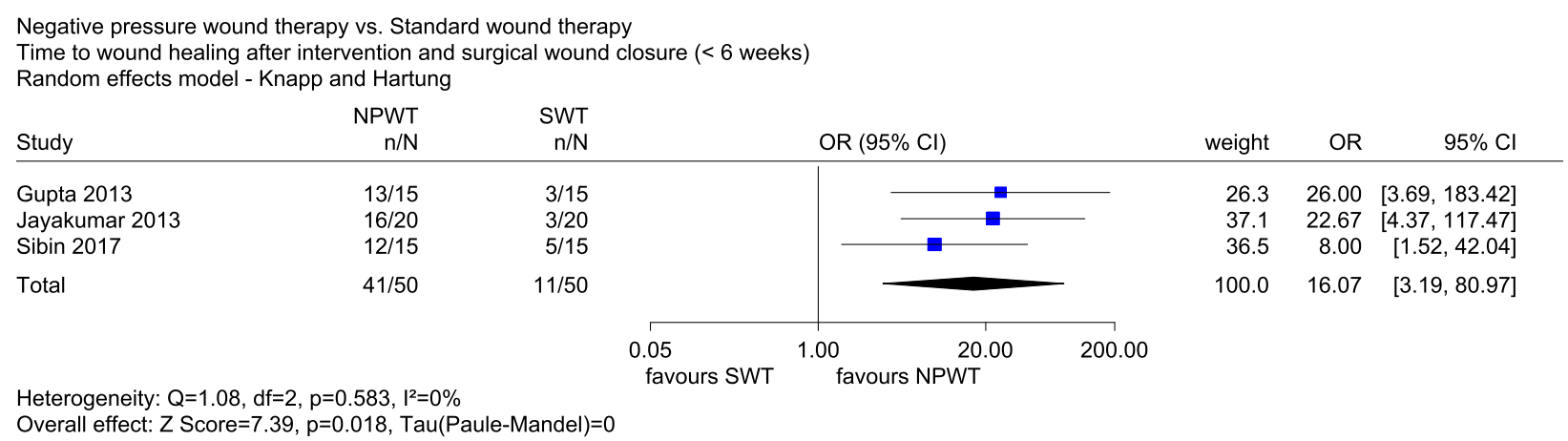

Fig. 4 Forest plot of time to wound healing after intervention and surgical wound closure ( $<6$ weeks) with overall effect estimation, NPWT vs. SWT. Abbreviations: Cl confidence interval, $n$ number of events, $N$ number of patients, NPWT negative pressure wound therapy, OR odds ratio, SWT standard wound therapy

the zero effect ( $\mathrm{OR}=1$ and Hedges' $\mathrm{g}=0)$; see Fig. 1 and 2 in Additional file 3. The heterogeneity for time to wound healing and/or surgical wound closure (in days) was mainly caused by Perez 2010. In a sensitivity analysis, the result of this study was shifted closer to zero, reducing heterogeneity. The mean value in the NPWT group was shifted towards the mean value in the SWT group until the test of heterogeneity was non-significant $(p>0.05)$. The resulting pooled effect was statistically significant (Hedges' g - 0.39, $95 \% \mathrm{CI}-0.66$ to $-0.11, p$ $=0.013$ ) but not clinically relevant, as the upper limit of the 95\% CI was not below the irrelevance threshold of 0.2 . There was neither proof (nor indication nor hint) of a greater or smaller effect of NPWT on wound healing and/or surgical wound closure (yes/no) and time to wound healing and/or surgical wound closure (in days).

Overall, there was proof of a greater benefit of NPWT for wound closure. Due to the potential publication bias mentioned above, this conclusion was downgraded. There was thus an indication of a greater benefit of NPWT for wound closure.

Adverse events comprised additional measures required for direct wound closure (such as skin transplantation or sutures), re-interventions (such as regrafting or revision fixation), bleeding, infections, the overall rate of serious adverse events and study discontinuation due to adverse events.

Ten studies provided data on re-interventions (Chiang 2017, De Laat 2011, Liao 2012, Llanos 2006, Mohsin 2017, Moisidis 2004, Saaiq 2010, VAC 2001-07, VAC 2001-08 and WOLLF). One study with a low risk of bias (Llanos 2006) showed no statistically significant difference between groups. In the combined analysis of studies with a low and high risk of bias, NPWT significantly reduced the odds for re-interventions (OR 0.46, 95\% CI 0.24 to $0.86, p=0.021$, see Fig. 3 in Additional file 3). One study (Perez 2010) measured the number of operations until wound closure and found a statistically significant difference in favour of SWT (MD 2.80, 95\% CI 0.79 to $4.81, p=0.008$ ). Due to the small number of patients in this study, the result of the meta-analysis was not challenged. There was thus an indication of a greater effect of NPWT on re-intervention.

There were no statistically significant differences between groups with regard to additional measures required for direct wound closure (23 studies, see Fig. 4 in

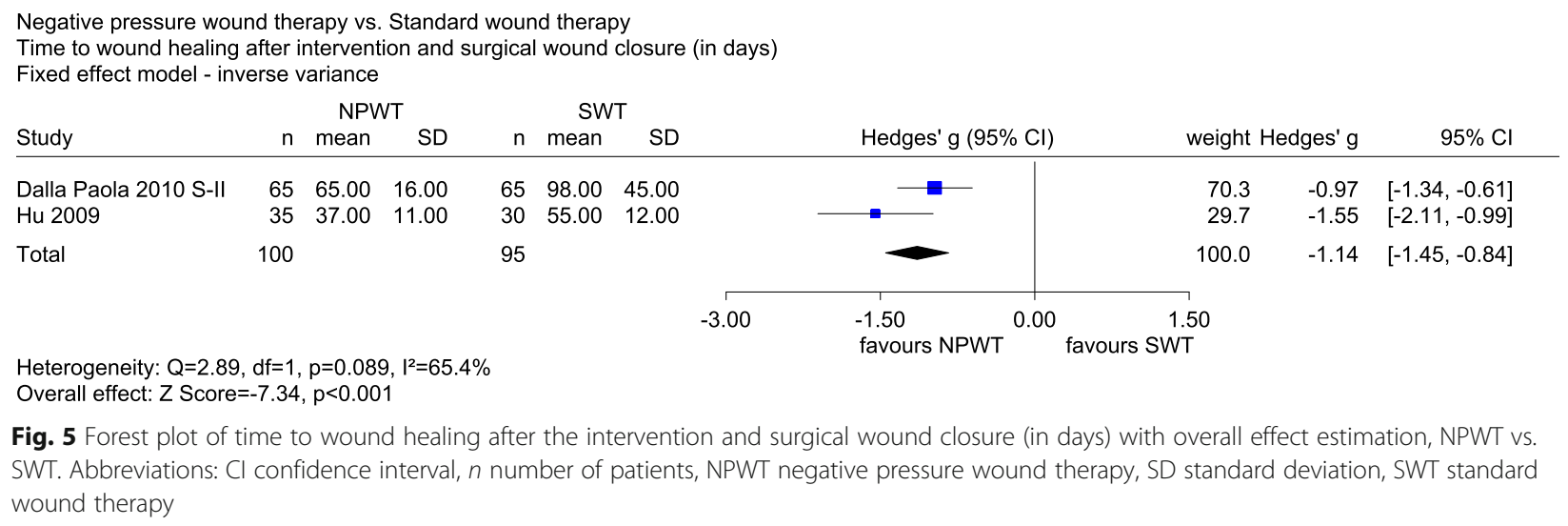

Fig. 5 Forest plot of time to wound healing after the intervention and surgical wound closure (in days) with overall effect estimation, NPWT vs. SWT. Abbreviations: Cl confidence interval, $n$ number of patients, NPWT negative pressure wound therapy, SD standard deviation, SWT standard wound therapy 


\begin{tabular}{|c|c|c|c|c|c|c|c|c|c|c|c|}
\hline \multirow{2}{*}{$\begin{array}{l}\text { Study pool } \\
\text { Study }\end{array}$} & \multirow[b]{2}{*}{$\mathrm{n}$} & \multicolumn{2}{|c|}{ NPWT } & \multicolumn{3}{|c|}{ SWT } & \multirow{2}{*}{\multicolumn{2}{|c|}{ mean difference $(95 \% \mathrm{Cl})$}} & \multirow{2}{*}{\multicolumn{2}{|c|}{$\begin{array}{r}\text { mean } \\
\text { weight difference }\end{array}$}} & \multirow[b]{2}{*}{$95 \% \mathrm{C}$} \\
\hline & & mean & SD & $\mathrm{n}$ & mean & SD & & & & & \\
\hline \multicolumn{12}{|l|}{ Risk of bias: low } \\
\hline Llanos $2006^{*}$ & 30 & 13.50 & 2.70 & 30 & 17.00 & 5.15 & $\rightarrow$ & & 100.0 & -3.50 & {$[-5.58,-1.42]$} \\
\hline \multicolumn{12}{|l|}{ Risk of bias: low + high } \\
\hline Acosta $2013^{*}$ & 10 & 13.00 & 28.45 & 10 & 20.00 & 22.63 & $\leftarrow$ & & 1.3 & -7.00 & {$[-29.53,15.53]$} \\
\hline Huang $2006^{*}$ & 12 & 32.10 & 14.07 & 12 & 34.30 & 11.93 & $=$ & & 5.6 & -2.20 & {$[-12.63,8.23]$} \\
\hline Liao 2012 & 30 & 12.00 & 14.24 & 30 & 18.00 & 17.53 & $=$ & - & 8.7 & -6.00 & {$[-14.08,2.08]$} \\
\hline Llanos $2006^{*}$ & 30 & 13.50 & 2.70 & 30 & 17.00 & 5.15 & $=$ & & 34.9 & -3.50 & {$[-5.58,-1.42]$} \\
\hline Rencüzogullari 2015 & 15 & 28.50 & 21.30 & 13 & 27.40 & 25.30 & & 1 & 2.2 & 1.10 & {$[-16.37,18.57]$} \\
\hline TOPSKIN w ds* & 21 & 21.00 & 10.85 & 23 & 24.00 & 15.04 & $=$ & - & 9.3 & -3.00 & {$[-10.70,4.70]$} \\
\hline TOPSKIN w/o ds* & 22 & 22.00 & 18.59 & 20 & 21.00 & 12.31 & & $=$ & 6.7 & 1.00 & {$[-8.46,10.46]$} \\
\hline VAC $2001-06^{*}$ & 35 & 9.50 & 16.29 & 23 & 11.70 & 16.29 & & & 7.9 & -2.20 & {$[-10.77,6.37]$} \\
\hline VAC 2001-07 & 60 & 25.00 & 31.29 & 64 & 26.94 & 32.28 & & & 5.0 & -1.94 & {$[-13.13,9.25]$} \\
\hline Vuerstaek $2006^{*}$ & 30 & 29.00 & 9.78 & 30 & 45.00 & 24.59 & $\longleftarrow$ & & 6.7 & -16.00 & {$[-25.47,-6.53]$} \\
\hline Xu 2015 & 20 & 21.00 & 8.50 & 20 & 32.00 & 12.52 & $\longrightarrow$ & & 11.7 & -11.00 & {$[-17.63,-4.37]$} \\
\hline REM - Knapp-Hartung & & & & & & & & & & -4.78 & {$[-7.79,-1.76]$} \\
\hline \multicolumn{12}{|c|}{$\begin{array}{l}\text { Heterogeneity: } Q=12.80, d f=10, p=0.235, I^{2}=21.9 \% \\
\text { Overall effect: } Z \text {-Score }=-3.53, p=0.005, \text { Tau(Paule-Mandel })=2.026\end{array}$} \\
\hline & & & & & & & $\begin{array}{ll}-20.00 & -10.00 \\
\text { favours NPWT }\end{array}$ & $.00 \begin{array}{r}10.00 \\
\text { favours SW }\end{array}$ & 20.00 & & \\
\hline
\end{tabular}

Fig. 6 Forest plot of hospital stay (in days) with overall effect estimation, NPWT vs. SWT. Abbreviations: Cl confidence interval, $n$ number of patients, NPWT negative pressure wound therapy, SD standard deviation, SWT standard wound therapy

Additional file 3) and the overall rate of serious adverse events (12 studies, see Fig. 5 in Additional file 3). No effect estimate was calculated for infection (20 studies, see Fig. 6 in Additional file 3) and study discontinuation due to adverse events (7 studies, see Fig. 7 in Additional file 3) because of heterogeneity. In these 2 analyses, the 95\% PI included the zero effect $(\mathrm{OR}=1)$. For bleeding (5 studies, see Fig. 8 in Additional file 3), no events occurred in 3 out of 6 studies. The other 3 studies showed no statistically significant differences. No overall effect was therefore calculated. There was neither proof (nor indication nor hint) of a greater or smaller effect of NPWT on any of these outcomes. As the overall rate of serious adverse events was the primary analysis for adverse events, there was neither proff (nor indication nor hint) of greater benefit or harm of NPWT for adverse events.

Hospital stay and readmission was measured as hospital stay (in days) in 10 studies (Acosta 2013, Huang 2006, Liao 2012, Llanos 2006, Rencüzoğulları 2015, TOPSKIN, VAC 2001-06, VAC 2001-07, Vuerstaek 2006 and $\mathrm{Xu}$ 2015), hospital stay (> 1 month yes/no) in 4 studies (Gupta 2013, Jayakumar 2013, Saaiq 2010 and

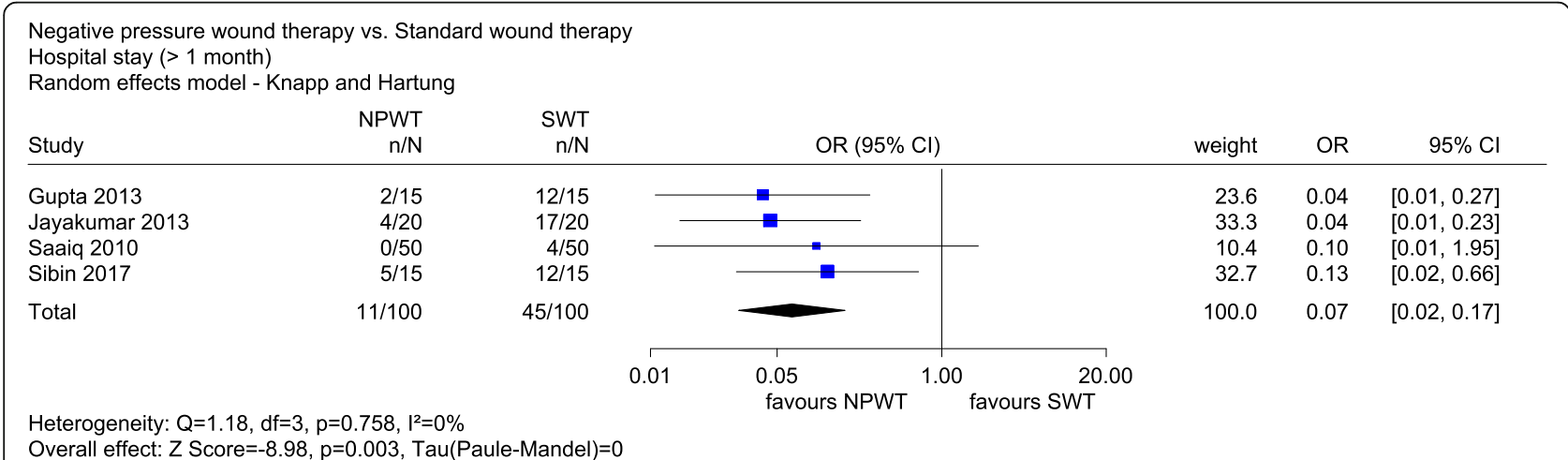

Fig. 7 Forest plot of hospital stay (> 1 month) with overall effect estimation, NPWT vs. SWT. Abbreviations: Cl confidence interval, $n$ number of events, N number of patients, NPWT negative pressure wound therapy, OR odds ratio, SWT standard wound therapy 
Sibin 2017), intensive care unit stay (in days) in 2 studies (Rencüzoğulları 2015 and TOPSKIN) and readmission (yes/no) in 4 studies (CE/044/PIC, De Laat 2011, SWHSI and VAC 2001-07). For all outcomes and studies (except one [Llanos 2006]), the risk of bias was high.

For hospital stay (in days), the study with a low risk of bias (MD -3.50 , 95\% CI -5.58 to -1.42 , see Fig. 6), as well as studies with a high and low risk of bias (MD 4.78, 95\% CI -7.79 to $-1.76, p=0.005$, see Fig. 6) showed a statistically significant difference in favour of NPWT. There was a proof of a greater effect of NPWT on hospital stay (in days).

Hospital stay (> 1 month yes/no) also showed a statistically significant effect in favour of NPWT (OR 0.07, 95\% CI 0.02 to $0.17, p=0.003$, see Fig. 7). There was an indication of a greater effect of NPWT on hospital stay (> 1 month).

Despite homogeneity between the studies $\left(I^{2}=0 \%, p=\right.$ 0.441), no effect estimate was presented for intensive care unit stay (in days) because of the large range of the $95 \% \mathrm{CI}$ (containing the zero effect MD =0). There was no statistically significant effect for readmission (yes/no) (OR 1.01, 95\% CI 0.44 to 2.31, $p=0.973$, see Fig. 9 in Additional file 3). There was neither proof (nor indication nor hint) of a greater or smaller effect of NPWT on the 2 latter outcomes.

Overall, there was proof of a greater benefit of NPWT for hospital stay and readmission. Due to the high risk of publication bias, the evidence for this outcome was downgraded. There was thus an indication of a greater benefit of NPWT for hospital stay and readmission.

Data on mortality were available in 18 studies (Acosta 2013, Ashby 2012, Bee 2018, Braakenburg 2006, Correa 2016, DiaFu, Huang 2006, Mouës 2004, Rencüzogullari 2015, Saaiq 2010, VAC 2001-01, VAC 2001-07, VAC 2001-08, VAC 2002-09, VAC 2002-10, Vuerstaek 2006, WOLLF and Xu 2015). There was no statistically significant effect of NPWT compared to SWT (OR 1.20, $95 \%$ CI 0.84 to $1.70, p=0.290$, see Fig. 10 in Additional file 3).

Data on amputation were available in 10 studies (Acosta 2013, Braakenburg 2006, Dalla Paola 2010 S-II, DiaFu, Hu 2009, Huang 2006, Mody 2008, SWHSI, VAC 2001-06 and WOLLF). There was no statistically significant effect of NPWT compared to SWT (OR 0.89, 95\% CI 0.55 to $1.43, p=0.588$, see Fig. 11 in Additional file 3).

Pain was measured continuously in 6 studies (visual analogue scale [Banasiewicz 2013, Biter 2014, SWHSI and Vuerstaek 2006], visual analogue thermometer [TOPSKIN], numeric rating scale [DiaFu]), dichotomously (pain yes/no) in 3 studies (Ashby 2012, Mody 2008 and WOLLF), and as pain on application and removal of dressing (continuously [SWHSI], dichotomously [CE/044/ PIC]). None of these outcomes showed a statistically significant difference between NPWT and SWT (see Figs. 12 and 13 and eTable 1 and eTable 2 in Additional file 3).
Two studies reported data on health-related quality of life using the Physical Composite Scale (PCS) and the Mental Health Composite Scale (MCS) derived from the SF-12 after 3 months (SWHSI) and 12 months (WOLLF), respectively. There was no statistically significant difference between NPWT and SWT for the MCS; heterogeneity was shown for the PCS (see Figs. 14 and 15 in Additional file 3).

Three studies provided data on physical function measured as time to resume work or school (Biter 2004), time to restoration of normal activity (Banasiewicz 2013) and the Disability Rating Index (DRI; [WOLLF]). A meta-analysis of the first 2 outcomes showed heterogeneity, while the DRI showed no statistically significant difference between NPWT and SWT (see Fig. 16 and eTable 3 in Additional file 3).

No study reported data on dependence on outside help or need for care. There was neither proof (nor indication nor hint) of a greater benefit or harm of NPWT for mortality, amputation, health-related quality of life, physical function and dependence on outside help or need for care.

Using the beta-binomial model to account for doublezero studies did not alter the results of the metaanalyses presented.

A summary of the results is presented in Table 5. An overview of the key findings according to GRADE methods can be found in Additional file 4.

\section{Discussion}

\section{Summary of findings}

This systematic review of NPWT versus SWT in patients with wounds healing by secondary intention showed some advantages of NPWT with regard to wound closure and hospital stay. No differences were shown for any other important outcomes such as infection or amputation (see Table 5).

\section{Comparison with previous research}

It is difficult to compare the present results with previous systematic reviews on NPWT due to their more or less restricted focus, in contrast to the rather wide question of the present review, which is wounds healing by secondary intention. Using only publications from 2013 to 2018, we identified 30 systematic reviews on NPWT for various wounds healing by secondary intention (see Additional file 5). Only 13 analysed wound closure, the key outcome for this type of intervention. Of these, 10 came to a positive conclusion [163-172], while 3 did not [173-175]. More importantly, only 14 [163-166, 168, $169,171,173,175-180]$ of the 30 previous systematic reviews at least mentioned the risk of publication bias and none implemented any consequences for their conclusions in the event of this type of bias. 
Table 5 Summary of results

\begin{tabular}{|c|c|c|}
\hline Outcome & Results & $\begin{array}{l}\text { Grading of } \\
\text { results }\end{array}$ \\
\hline Mortality & OR $1.20,95 \% \mathrm{Cl} 0.84$ to $1.70, p=0.290$ & $\Leftrightarrow$ \\
\hline \multicolumn{2}{|l|}{ Wound closure } & \multirow[t]{2}{*}{$\Uparrow^{a}$} \\
\hline Wound healing (yes/no) & OR $1.56,95 \% \mathrm{Cl} 1.15$ to $2.13, p=0.008$ & \\
\hline Time to wound healing (in days) & $\begin{array}{l}\text { Studies with low risk of bias: } \\
\text { Hedges' } \mathrm{g}-1.33,95 \% \mathrm{Cl}-1.90 \text { to }-0.77 \\
\text { Studies with low and high risk of bias: } \\
\text { Hedges' } \mathrm{g}-0.77,95 \% \mathrm{Cl}-1.19 \text { to }-0.35, p=0.005\end{array}$ & $\Uparrow \Uparrow$ \\
\hline $\begin{array}{l}\text { Time to wound healing after intervention and } \\
\text { surgical wound closure }\end{array}$ & $\begin{array}{l}\text { < } 6 \text { weeks yes/no: } \\
\text { OR 16.07, } 95 \% \mathrm{Cl} 3.19 \text { to } 80.97, p=0.018 \\
\text { In days: } \\
\text { Hedges' } \mathrm{g}-1.14,95 \% \mathrm{Cl}-1.45 \text { to }-0.84, p<0.001\end{array}$ & $\Uparrow$ \\
\hline $\begin{array}{l}\text { Wound healing and/or surgical wound } \\
\text { closure (yes/no) }\end{array}$ & $\begin{array}{l}\text { Heterogeneous effects (OR) } \\
95 \% \text { PI } 0.37 \text { to } 4.97\end{array}$ & $\Leftrightarrow$ \\
\hline $\begin{array}{l}\text { Time to wound healing and/or surgical } \\
\text { wound closure (in days) }\end{array}$ & $\begin{array}{l}\text { Heterogeneous effects (Hedges' g) } \\
95 \% \mathrm{PI}-3.47 \text { to } 2.10\end{array}$ & $\Leftrightarrow$ \\
\hline \multicolumn{2}{|l|}{ Adverse events } & \multirow[t]{2}{*}{$\Leftrightarrow^{b}$} \\
\hline $\begin{array}{l}\text { Additional measures required for direct } \\
\text { wound closure }\end{array}$ & OR $1.20,95 \% \mathrm{Cl} 0.70$ to $2.08, p=0.476$ & \\
\hline Re-interventions & $\begin{array}{l}\text { Yes/no: } \\
\text { OR } 0.46,95 \% \text { Cl } 0.24 \text { to } 0.86, p=0.021 \\
\text { Number of re-interventions: } \\
\text { MD } 2.80,95 \% \text { Cl } 0.79 \text { to } 4.81, p=0.008\end{array}$ & $\pi^{c}$ \\
\hline Bleeding & $\begin{array}{l}\text { No effect estimate (OR) is given due to } 3 \text { out of } 6 \text { studies without events and } 3 \\
\text { studies with no statistically significant effects. }\end{array}$ & $\Leftrightarrow$ \\
\hline Infection & $\begin{array}{l}\text { Heterogeneous effects (OR) } \\
95 \% \text { PI } 0.07 \text { to } 5.21\end{array}$ & $\Leftrightarrow$ \\
\hline Overall rate of SAES & OR $1.02,95 \% \mathrm{Cl} 0.76$ to $1.37, p=0.860$ & $\Leftrightarrow$ \\
\hline Study discontinuation due to AEs & $\begin{array}{l}\text { Heterogeneous effects (OR) } \\
95 \% \mathrm{PI} 0.36 \text { to } 13.57\end{array}$ & $\Leftrightarrow$ \\
\hline Amputation & OR $0.89,95 \% \mathrm{Cl} 0.55$ to $1.43, p=0.588$ & $\Leftrightarrow$ \\
\hline \multicolumn{2}{|l|}{ Pain } & $\Leftrightarrow$ \\
\hline Continuous & Hedges' $\mathrm{g}-0.16,95 \% \mathrm{Cl}-0.53$ to $0.21, p=0.32$ & $\Leftrightarrow$ \\
\hline Dichotomous & $\begin{array}{l}\text { No effect estimate }(O R) \text { is given due to the large range of the } 95 \% \mathrm{Cl} \text { containing } \\
\text { the zero effect }(\mathrm{OR}=1) \text {. }\end{array}$ & $\Leftrightarrow$ \\
\hline Pain on application and removal of dressing & $\begin{array}{l}\text { Continuous: } \\
\text { MD - 0.30, } 95 \% \mathrm{Cl}-19.75 \text { to } 19.15, p=0.975 \\
\text { Dichotomous: } \\
\text { No statistically significant effects (OR) for all given weeks }\end{array}$ & $\Leftrightarrow$ \\
\hline \multicolumn{2}{|l|}{ Hospital stay and readmission } & \multirow[t]{2}{*}{$\Uparrow^{a}$} \\
\hline Hospital stay (in days) & $\begin{array}{l}\text { Study with low risk of bias: } \\
\mathrm{MD}-3.50,95 \% \mathrm{Cl}-5.58 \text { to }-1.42 \\
\text { Studies with low and high risk of bias: } \\
\mathrm{MD}-4.78,95 \% \mathrm{Cl}-7.79 \text { to }-1.76, p=0.005\end{array}$ & \\
\hline Hospital stay (> 1 month yes/no) & OR $0.07,95 \% \mathrm{Cl} 0.02$ to $0.17, p=0.003$ & $\Uparrow$ \\
\hline Intensive care unit stay (in days) & $\begin{array}{l}\text { No effect estimate }(M D) \text { is given due to the large range of the } 95 \% \mathrm{Cl} \text { containing } \\
\text { the zero effect }(M D=0) \text {. }\end{array}$ & $\Leftrightarrow$ \\
\hline Readmission (yes/no) & OR $1.01,95 \% \mathrm{Cl} 0.44$ to $2.31, p=0.973$ & $\Leftrightarrow$ \\
\hline \multicolumn{2}{|l|}{ Health-related quality of life } & $\Leftrightarrow$ \\
\hline SF-12 MCS & Hedges' g $0.01,95 \% \mathrm{Cl}-0.20$ to $0.22, p=0.937$ & $\Leftrightarrow$ \\
\hline SF-12 PCS & Heterogeneous effects (Hedges' g) & $\Leftrightarrow$ \\
\hline Physical function & & $\Leftrightarrow$ \\
\hline Time to resume work or school/restoration of & Heterogeneous effects (Hedges' g) & $\Leftrightarrow$ \\
\hline
\end{tabular}


Table 5 Summary of results (Continued)

\begin{tabular}{|c|c|c|}
\hline Outcome & Results & $\begin{array}{l}\text { Grading of } \\
\text { results }\end{array}$ \\
\hline \multicolumn{3}{|l|}{ normal activity (in days) } \\
\hline DRI & No statistically significant effects (MD) for all given months & $\Leftrightarrow$ \\
\hline $\begin{array}{l}\text { Dependence on outside help or need for } \\
\text { care }\end{array}$ & No data & $\Leftrightarrow$ \\
\hline \multicolumn{3}{|c|}{$\begin{array}{l}A E \text { adverse event, } C I \text { confidence interval, DRI Disability Rating Index, } M C S \text { mental health composite scale, } M D \text { mean difference, NWPT negative pressure wound } \\
\text { therapy, } O R \text { odds ratio, } p p \text { value, } P C S \text { physical composite scale, } P I \text { prediction interval, SAE serious adverse event, SF short for } \\
\text { aThe proof was downgraded due to high risk of publication bias } \\
\text { bThe overall rate of SAEs was the primary analysis of adverse events } \\
\text { 'The indication of a greater effect of NWPT measured as re-intervention (yes/no) was not challenged by the hint of a smaller effect of NWPT measured as number }\end{array}$} \\
\hline
\end{tabular}

\section{Data pooling}

The question as to whether to pool clinical study data or not is fundamental in meta-analysis. In the assessment of NPWT, various medical aspects need to be considered before pooling the data into a common effect estimate. The type of wound investigated is the most obvious difference between the studies in our analysis. Further factors potentially affecting study results are the exact type of NPWT technique used (e.g. pressure applied), choice of control treatment, type of healthcare setting and study duration. However, against the background of the lack of a standardised nomenclature for wounds and the fact that if the underlying disease and the respective wound is prepared optimally, wounds are very similar, it seemed meaningful to pool the data with regard to the type of wound healing. Furthermore, innovation would become impossible if each new wound treatment had to be tested for each of the numerous different wound types. Since several meta-analyses showed no or only little heterogeneity, our data at least do not contradict this approach.

Published evidence tends to overestimate the benefits and underestimate the harms of medical interventions [181], and it is widely accepted that the results of all relevant studies must be fully available for an unbiased estimation of effects. The introduction of mandatory measures such as registration of studies and their results has increased data availability, but many studies (in the present case mostly IITs), especially on medical devices, still remain partly or fully unpublished. Further measures to ensure full disclosure are thus urgently required. As the present case shows that voluntary commitment does not work, further legal and regulatory action, in combination with sanctions, seems to be indispensable. For instance, IQWiG proposes that funders of clinical research and ethic committees exert stronger supervision over research projects by denying further funding if previous projects were not properly published [18].

\section{Strengths and limitations}

Strengths of the present research include the systematic literature search and the inclusion of several unpublished studies, which were in particular obtained from several NPWT manufacturers (and sometimes provided only after public pressure). This methodological rigour was essential in order to limit potential publication bias. Nevertheless, the present review included an insufficient proportion of the relevant clinical study data to draw highly robust conclusions, which is why our conclusions are cautious. In addition, we did not assess outcome reporting bias. We cannot exclude that the proportion of missing data would have been even higher if this type of bias had also been considered. It should also be noted that overall, the quality of the studies considered was low and the sample sizes were small. If larger, independently funded, multicentre trials had been performed, evaluation of NPWT would have been possible in an earlier, easier and more reliable way.

\section{Implications for future research}

The advantages of NPWT were modest, and the main conclusion with regard to wound closure was derived from only 14 of the 48 studies. Due to various definitions of wound closure and different time points of data collection (or even missing information on these items), no conclusions can be made with regard to the sustainability of wound closure. In addition, as stated, the size and quality of the studies were generally low. Therefore, it is certainly not unethical to conduct further (but highquality) RCTs on this topic. We recommend that they systematically investigate and clearly define the key outcomes of wound healing, adverse events and healthrelated quality of life.

\section{Conclusion}

In summary, low-quality data indicate a greater benefit of NPWT versus SWT for the outcome of wound 
closure in patients with wounds healing by secondary intention. The length of hospital stay is also shortened. However, the data show no advantages or disadvantages of NPWT versus SWT for mortality, adverse events, amputation, pain or health-related quality of life. Although data on serious adverse events were not systematically collected in most of the primary studies, NPWT appears to be safe. Publication bias is an important problem in NPWT research, underlining that all clinical studies need to be fully reported regardless of funding source, premature study termination or study results.

\section{Supplementary information}

Supplementary information accompanies this paper at https://doi.org/10. 1186/s13643-020-01476-6.

Additional file 1. Search strategies applied and manufacturers contacted (DOCX $27 \mathrm{~kb}$ )

Additional file 2. Detailed risk of bias for assessments of outcomes (DOCX 49 kb)

Additional file 3. Forest plots not presented in the manuscript (DOCX $62 \mathrm{~kb}$ )

Additional file 4. Overview of key findings according to GRADE (DOCX $18 \mathrm{~kb})$

Additional file $\mathbf{5}$. List of previous systematic reviews (DOCX $18 \mathrm{~kb}$ )

\section{Abbreviations}

Cl: Confidence interval; CSR: Clinical study report; DRI: Disability Rating Index; HTA: Health technology assessment; IIT: Investigator-initiated trial; MCS: Mental Health Composite Scale; MD: Mean difference; NPWT: Negative pressure wound therapy; OR: Odds ratio; PI: Prediction interval; PCS: Physical Composite Scale; PRISMA: Preferred Reporting Items for Systematic Reviews and Meta-Analyses; RCT: Randomised controlled trial; SWT: Standard wound therapy

\section{Acknowledgements}

The German Institute for Quality and Efficiency in Health Care supported this work. No external funding was received. The authors thank Siw Waffenschmidt for her support in developing and conducting the literature search, Heike Raatz for her contribution to literature screening and Natalie McGauran for editorial support.

\section{Authors' contributions}

YZ wrote the main part of the manuscript, made substantial contributions to the study design and was involved in screening and in the collection and interpretation of data. MB was involved in the screening, the collection and interpretation of data, and in writing the manuscript. HB provided methodological expertise and was involved in writing the manuscript. KD was involved in the collection of data and in writing the manuscript. MF made substantial contributions to the study design, performed the statistical analyses, and was involved in the analysis and interpretation of data, and in writing the manuscript. WG was involved in the collection of data and in writing the manuscript. TJ was involved in the collection and interpretation of data, as well as in writing the manuscript. HK was involved in the collection and interpretation of data, as well as in writing the manuscript. MK performed the statistical analyses and was involved in the analysis and interpretation of data, as well as in writing the manuscript. IO developed and conducted the literature search and was involved in writing the manuscript. StS made substantial contributions to the study design and was involved in the interpretation of data and in writing the manuscript. SG provided clinical expertise and was involved in screening and in the interpretation of data and in writing the manuscript. All authors read and approved the final version of the manuscript.

\section{Funding}

Open Access funding enabled and organized by Projekt DEAL.

\section{Availability of data and materials}

All data used in this article are available in the full German-language report published on the IQWiG website [9].

\section{Competing interests}

SG and the research institute of $\mathrm{HB}$ each received an honorarium from IQWiG for their contributions to the HTA that formed the basis for this publication. The other authors declare no competing interests.

\section{Author details}

${ }^{1}$ Institute for Quality and Efficiency in Health Care (IQWiG), Im Mediapark 8, 50670 Cologne, Germany. ${ }^{2}$ Basel Institute for Clinical Epidemiology \& Biostatistics, University Hospital Basel and University of Basel, Basel, Switzerland. ${ }^{3}$ Düsseldorf, Germany.

Received: 5 September 2019 Accepted: 7 September 2020

Published online: 10 October 2020

\section{References}

1. Graham ID, Harrison MB, Nelson EA, Lorimer K, Fisher A. Prevalence of lower-limb ulceration: a systematic review of prevalence studies. Adv Skin Wound Care 2003; 16(6): 305-316.

2. Kaltenthaler E, Whitfield MD, Walters SJ, Akehurst RL, Paisley S. UK, USA and Canada: how do their pressure ulcer prevalence and incidence data compare? J Wound Care 2001; 10(1): 530-535.

3. Wu S, Armstrong DG. Risk assessment of the diabetic foot and wound. Int Wound J 2005; 2(1): 17-24.

4. Persoon A, Heinen MM, van der Vleuten CJ, de Rooij MJ, van de Kerkhof PC, van Achterberg T. Leg ulcers: a review of their impact on daily life. J Clin Nurs 2004; 13(3): 341-354.

5. Argenta LC, Morykwas MJ. Vacuum-assisted closure: a new method for wound control and treatment: clinical experience. Ann Plast Surg 1997; 38(6): 563-576; discussion 577.

6. Institut für Qualität und Wirtschaftlichkeit im Gesundheitswesen. Vakuumversiegelungstherapie von Wunden: Abschlussbericht; Auftrag N0403 [online]. 13.03.2006 [Zugriff: 11.03.2013]. (IQWiG-Berichte; Band 4). URL: http://www.iqwig.de/download/N04-03_Abschlussbericht_ Vakuumversiegelungstherapie_zur_Behandlung_von_Wunden..pdf.

7. Institut für Qualität und Wirtschaftlichkeit im Gesundheitswesen. Vakuumversiegelungstherapie von Wunden: rapid report; Auftrag N06-02 [online]. 29.01.2007 [Zugriff: 11.03.2013]. (IQWiG-Berichte; Band 16). URL: http://www.iqwig.de/download/N06-02_Rapid_Report_ Vakuumversiegelungstherapie_von_Wunden..pdf.

8. Gregor S, Maegele M, Sauerland S, Krahn JF, Peinemann F, Lange S. Negative pressure wound therapy: a vacuum of evidence? Arch Surg 2008: 143(2): 189-196

9. Institute for Quality and Efficiency in Health Care. [N17-01A] Vakuumversiegelungstherapie von Wunden mit intendierter sekundärer Wundheilung [online]. [Zugriff: 13.06.2019]. URL: https://www.iqwig.de/de/ projekte-ergebnisse/projekte/nichtmedikamentoese-verfahren/n-projekte/ n17-01a-vakuumversiegelungstherapie-von-wunden-mit-intendiertersekundaerer-wundheilung.9654.html.

10. Institut für Qualität und Wirtschaftlichkeit im Gesundheitswesen. Vakuumversiegelungstherapie von Wunden: vorläufiger Berichtsplan; Auftrag N17-01 [online]. 16.06.2017 [Zugriff: 05.07.2017]. URL: https://www. iqwig.de/download/N17-01_Vakuumversiegelungstherapie-von-Wunden_ Vorlaeufiger-Berichtsplan_V1-0.pdf.

11. Moher D, Liberati A, Tetzlaff J, Altman DG, Group P. Preferred reporting items for systematic reviews and meta-analyses: the PRISMA statement. Int J Surg 2010; 8(5): 336-341.

12. Biomarkers Definitions Working Group. Biomarkers and surrogate endpoints: preferred definitions and conceptual framework. Clinical Pharmacology and Therapeutics 2001; 69(3): 89-95.

13. Wan X, Wang W, Liu J, Tong T. Estimating the sample mean and standard deviation from the sample size, median, range and/or interquartile range. BMC Medical Research Methodology 2014; 14: 135.

14. Veroniki AA, Jackson D, Viechtbauer W, Bender R, Knapp G, Kuss $O$ et al. Recommendations for quantifying the uncertainty in the summary 
intervention effect and estimating the between-study heterogeneity variance in random-effects meta-analysis. Cochrane Database Syst Rev 2015: (Suppl 1): 25-27.

15. Borenstein M, Hedges LV, Higgins JPT, Rothstein HR. Introduction to MetaAnalysis. Chichester, U.K.: John Wiley \& Sons; 2009.

16. Kuss O. Statistical methods for meta-analyses including information from studies without any events: add nothing to nothing and succeed nevertheless. Stat Med 2015; 34(7): 1097-1116.

17. Higgins JPT, Thompson SG, Spiegelhalter DJ. A re-evaluation of randomeffects meta-analysis. Journal of the Royal Statistical Society Series A (Statistics in Society) 2009; 172(1): 137-159.

18. Institut für Qualität und Wirtschaftlichkeit im Gesundheitswesen. Vakuumversiegelungstherapie von Wunden mit intendierter sekundärer Wundheilung: Abschlussbericht; Auftrag N17-01A [online]. 24.01.2019 [Zugriff: 01.04.2019]. (IQWiG-Berichte; Band 713). URL: https://www.iqwig.de/ download/N17-01A_Abschlussbericht_Vakuumversiegelungstherapie-vonWunden-mit-inte....pdf.

19. Georgetown University. ActiV.A.C.+ compression therapy versus compression therapy alone for the treatment of chronic venous ulcerations: full text view [online]. In: ClinicalTrials.gov. 21.12.2016 [Zugriff: 30.08.2017]. URL: https://ClinicalTrials.gov/show/NCT01528293.

20. Dwivedi MK, Srivastava RN, Bhagat AK, Agarwal R, Baghel K, Jain A et al. Pressure ulcer management in paraplegic patients with a novel negative pressure device: a randomised controlled trial. Journal of Wound Care 2016; 25(4): 199-200

21. Dwivedi MK, Bhagat AK, Srivastava RN, Jain A, Baghel K, Raj S. Expression of MMP-8 in pressure injuries in spinal cord injury patients managed by negative pressure wound therapy or conventional wound care: a randomized controlled trial. J Wound Ostomy Continence Nurs 2017; 44(4): $1-7$.

22. Eginton MT, Brown KR, Seabrook GR, Towne JB, Cambria RA. A prospective randomized evaluation of negative-pressure wound dressings for diabetic foot wounds. Ann Vasc Surg 2003; 17(6): 645-649.

23. Ford CN, Reinhard ER, Yeh D, Syrek D, De Las Morenas A, Bergman SB et al. Interim analysis of a prospective, randomized trial of vacuum-assisted closure versus the healthpoint system in the management of pressure ulcers. Ann Plast Surg 2002; 49(1): 55-61.

24. University of Witten/Herdecke. Evaluate the efficacy of the treatment of iatrogenic subcutaneous abdominal wounds (ISAW) after surgery by application of negative pressure wound therapy (NPWT) in comparison to standard conventional wound therapy (SCWT) of the clinical routine (ISAW): full text view [online]. In: ClinicalTrials.gov. 17.05.2013 [Zugriff: 30.08.2017]. URL: https://ClinicalTrials.gov/show/NCT01611207.

25. University of Witten/Herdecke. Treatment of iatrogenic subcutaneous abdominal wounds (ISAW) after surgery [online]. In: ISRCTN Registry. 04.08. 2016 [Zugriff: 30.08.2017]. URL: http://isrctn.com/ISRCTN44577192.

26. Private Universität Witten/Herdecke. Randomisiert, kontrollierte klinische Studie zur Untersuchung der Effektivität der Behandlung von latrogenen Subkutanen Abdominellen Wundheilungsstörungen (ISAW) nach chirurgischem Eingriff unter Nutzung des Therapieprinzips der Negative Pressure Wound Therapy im Vergleich zur Standard Conventional Wound Therapy der klinischen Routine [online]. In: Deutsches Register Klinischer Studien. 27.01.2012 [Zugriff: 30.08.2017]. URL: http://www.drks.de/ DRKS00003498.

27. Private Universität Witten/Herdecke. Randomisiert, kontrollierte klinische Studie zur Untersuchung der Effektivität der Behandlung von iatrogenen subkutanen abdominellen Wundheilungsstörungen nach chirurgischem Eingriff unter Nutzung der Therapieprinzips der Negative Pressure Wound Therapy im Vergleich zur Standard Conventional Wound Therapy der klinischen Routine (ISAW): study ISAW; Abschlussbericht [unveröffentlicht]. 2015.

28. Joseph E, Hamori CA, Bergman S, Roaf E, Swann NF, Anastasi GW. A prospective randomized trial of vaccum-assisted closure versus standard therapy of chronic nonhealing wounds. Wounds 2000; 12(3): 60-67.

29. Keskin M, Karabekmez FE, Yilmaz E, Tosun Z, Savaci N. Vacuum-assisted closure of wounds and anxiety. Scandinavian Journal of Plastic and Reconstructive Surgery and Hand Surgery 2008; 42(4): 202-205.

30. Riaz MU, Khan MUR, Akbar A. Comparison of vacuum assisted closure versus normal saline dressing in healing diabetic wounds. Pakistan Journal of Medical \& Health Sciences 2010; 4(4): 308-313.

31. Sajid MT, Mustafa QUA, Shaheen N, Hussain SM, Shukr I, Ahmed M. Comparison of negative pressure wound therapy using vacuum-assisted closure with advanced moist wound therapy in the treatment of diabetic foot ulcers. J Coll Physicians Surg Pak 2015; 25(11): 789-793.

32. Sun JW, Sun JH, Zhang CC. Vacuum assisted closure technique for repairing diabetic foot ulcers: analysis of variance by using a randomized and doublestage crossover design [Chinesisch]. Journal of Clinical Rehabilitative Tissue Engineering Research 2007; 11(14): 8908-8911.

33. Vaidhya N, Panchal A, Anchalia MM. A new cost-effective method of NPWT in diabetic foot wound. Indian J Surg 2015; 77(Suppl 2): S525-S529.

34. Wanner MB, Schwarzl F, Strub B, Zaech GA, Pierer G. Vacuum-assisted wound closure for cheaper and more comfortable healing of pressure sores: a prospective study. Scand J Plast Reconstr Surg Hand Surg 2003; 37(1): 28-33.

35. Medical University of Silesia. Negative pressure therapy in wound healing [online]. In: Australian New Zealand Clinical Trials Registry. 20.01.2014 [Zugriff: 30.08.2017]. URL: http://www.anzctr.org.au/ACTRN12614000056695.aspx.

36. Laboratoires Brothier. Comparison of the efficacy, tolerance and cost of Algostéril vs negative pressure therapy in preparation for skin grafting following surgical excision [online]. In: ISRCTN Registry. 26.01.2017 [Zugriff: 30.08.2017]. URL: http://isrctn.com/ISRCTN60292377.

37. VA Office of Research and Development. Investigation of subatmospheric pressure dressing on pressure ulcer healing: study details [online]. In: ClinicalTrials.gov. 09.02.2009 [Zugriff: 25.06.2018]. URL: https://clinicaltrials. gov/ct2/show/NCT00011531.

38. Jipmer. Comparison of vacuum assisted dressing and normal dressing in healing of diabetic foot ulcer [online]. In: Clinical Trials Registry India. 23.01. 2018 [Zugriff: 30.07.2018]. URL: http://www.ctri.nic.in/Clinicaltrials/pmaindet2. php?trialid=19771.

39. Dept. of Endocrinology \& Metabolism, Nanfang Hospital, Southern Medical University. A prospective multicenter assessment of Foryou NPWT security and effectiveness in promoting the healing of diabetic foot ulcer [online]. In: Chinese Clinical Trial Registry. 16.12.2015 [Zugriff: 30.08.2017]. URL: http:// www.chictr.org.cn/showproj.aspx?proj=6852.

40. Instituto Mexicano del Seguro Social. Vacuum assisted closure in neck abscess: study details [online]. In: ClinicalTrials.gov. 22.04.2014 [Zugriff: 26.07. 2018]. URL: https://ClinicalTrials.gov/show/NCT02102685.

41. Department of burn plastic surgery, the first hospital of Shijiazhuang city. Evaluation of cupping therapy for certain chronic wounds: a randomized controlled clinical trial of cupping therapy versus negative pressure wound therapy [online]. In: Chinese Clinical Trial Registry. 14.03.2016 [Zugriff: 30.08. 2017]. URL: http://www.chictr.org.cn/showproj.aspx?proj=13345.

42. The University of Texas Health Science Center at San Antonio. Study to determine the utility of wound vacuum assisted closure (VAC) compared to conventional saline dressing changes: study details [online]. In: ClinicalTrials. gov. 08.10.2015 [Zugriff: 26.07.2018]. URL: https://ClinicalTrials.gov/show/ NCT00121537.

43. Karolinska Institutet. Negative pressure wound therapy: therapy effects and the impact on the patient's quality of life: study details [online]. In: ClinicalTrials.gov. 17.11.2017 [Zugriff: 26.07.2018]. URL: https://ClinicalTrials. gov/show/NCT01191567.

44. Centre Hospitalier Universitaire de Saint Etienne. Comparison of the use of VAC system in transplants of leg ulcers versus usual dressing method: study details [online]. In: ClinicalTrials.gov. 28.07.2015 [Zugriff: 26.07.2018]. URL: https://ClinicalTrials.gov/show/NCT02509533.

45. $\mathrm{KCl}$. A controlled study comparing the effectiveness of subatmospheric pressure dressing to normal saline wet-to-moist dressing on pressure ulcers: study Greer 1999; study protocol [unveröffentlicht]. 1998.

46. KCl. Greer 1999 results [unveröffentlicht]. 1999.

47. St. Joseph's Healthcare Hamilton. Negative pressure wound therapy for the treatment of chronic pressure wounds (NPWT): study details [online]. In: ClinicalTrials.gov. 26.07.2011 [Zugriff: 26.07.2018]. URL: https://ClinicalTrials. gov/show/NCT00691821.

48. Armed Forces Hospital. Reducing the surgical site infection rate after loop colosromy reversal by application of vacuum assisted delayed wound closure: a randomized controlled trial [online]. In: ISRCTN Registry. 30.06 2017 [Zugriff: 30.08.2017]. URL: http://isrctn.com/ISRCTN37399763.

49. Farfus A. A randomised control trial pilot study for comparison of the single use negative pressure wound therapy device (NPWT) to current standard dressings on medium sized split skin grafts (SSG) for emergency and elective surgery cases to assess healing time/quality and of treatment costs [online]. In: Australian New Zealand Clinical Trials Registry. 25.07.2018 [Zugriff: 26.07.2018]. URL: http://www.anzctr. org.au/ACTRN12612000702819.aspx. 
50. Department of Health Victoria. Does negative pressure wound therapy (NPWT) increase healing rates in post-operative foot wounds in Hospital in the Home (HITH) patients [online]. In: Australian New Zealand Clinical Trials Registry. 20.08.2012 [Zugriff: 30.08.2017]. URL: http://www.anzctr.org.au/ ACTRN12612000885897.aspx.

51. Queensland Children's Medical Research Institute, University of Queensland. Examination of the effect of negative pressure wound therapy in acute paediatric burns on reepithelialisation, pain and injury progression: a pilot study [online]. In: Australian New Zealand Clinical Trials Registry. 07.10.2014 [Zugriff: 30.08.2017]. URL: http://www.anzctr.org.au/ACTRN12614001068651. aspx.

52. Wake Forest Baptist Medical Center. Evaluation of the efficacy of the V.A.C. on management of acute hand burns: study VAC 2001-00; study protocol [unveröffentlicht]. 2001

53. KCl. Burnvac study: study VAC 2001-00; interim analysis [unveröffentlicht]. 2003.

54. $\mathrm{KCl}$ USA. A study comparing V.A.C. negative pressure wound therapy (NPWT) to moist wound therapy (MWT) in the treatment of diabetic foot amputation wounds (VAC 2006-19): study details [online]. In: ClinicalTrials. gov. 14.10.2013 [Zugriff: 26.07.2018]. URL: https://ClinicalTrials.gov/show/ NCT00837096.

55. $\mathrm{KCl}$ USA. A prospective, randomized, multicenter, parallel study comparing V.A.C. negative pressure wound therapy (NPWT) to moist wound therapy (MWT) in the treatment of diabetic foot amputation wounds: study VAC 2006-19; clinical research protocol; version 1.10 [unveröffentlicht]. 2007.

56. $\mathrm{KCl}$ Medical Australia. Randomised controlled trial of the efficacy and costeffectiveness of the vacuum-assisted closure therapy (VAC) system as an alternative to standard arterial ulcer management in a hospital-at-home setting [online]. In: Australian New Zealand Clinical Trials Registry. 23.08.2018 [Zugriff: 07.11.2018]. URL: http://www.anzctr.org.au/ACTRN12606000384550. aspx.

57. $\mathrm{KCl}$. Randomised controlled trial of the efficacy and cost-effectiveness of the VAC system as an alternative to standard arterial ulcer management in a hospital-at-home setting: study VAC trial; protocol [unveröffentlicht]

58. First Affiliated Hospital, Sun Yat-Sen University. Negative pressure wound therapy for skin grafts: study details [online]. In: ClinicalTrials.gov. 02.03.2015 [Zugriff: 26.07.2018]. URL: https://ClinicalTrials.gov/show/NCT02374528.

59. Walter Reed National Military Medical Center. Pilonidal disease wound healing study: study details [online]. In: ClinicalTrials.gov. 20.05.2013 [Zugriff: 26.07.2018]. URL: https://ClinicalTrials.gov/show/NCT01857128.

60. Zhen ZJ. A randomized contolled trial of managing infected abdominal wound by continuous irrigation and negative pressure suction versus open wound dressings followed by secondary suturing [online]. In: Australian New Zealand Clinical Trials Registry. 16.03.2009 [Zugriff: 30.08.2017]. URL http://www.anzctr.org.au/ACTRN12609000149268.aspx.

61. Martin R. Wound outcomes in negative pressure dressings (WOUNDS): suction dressings and mobility compared to conventional dressings and bed rest for healing of split skin grafts of the lower leg [online]. In: Australian New Zealand Clinical Trials Registry. 04.08.2011 [Zugriff: 30.08. 2017]. URL: http://www.anzctr.org.au/ACTRN12609000995279.aspx.

62. Medical College Trivandrum. A clinical study comparing the effectiveness of vacuum assisted dressing and conventional management in cases of long standing diabetic ulcers [online]. In: Clinical Trials Registry India. 05.02.2014 [Zugriff: 30.08.2017]. URL: http://www.ctri.nic.in/Clinicaltrials/pmaindet2. php?trialid=8439.

63. Innovative Therapies. Efficacy of quantum NPWT with simultaneous irrigation on reduction of wound volume in stage III/IV pressure ulcers: study details [online]. In: ClinicalTrials.gov. 28.11.2012 [Zugriff: 26.07.2018]. URL: https://ClinicalTrials.gov/show/NCT01734109.

64. University Hospital Ghent. Negative pressure wound therapy and allogeneic human skin grafts for wound bed preparation (NPWTVSGPA): study details [online]. In: ClinicalTrials.gov. 11.12.2014 [Zugriff: 26.07.2018]. URL: https:// ClinicalTrials.gov/show/NCT02314468.

65. Acosta S, Monsen C, Dencker M. Clinical outcome and microvascular blood flow in VAC- and Sorbalgon -treated peri-vascular infected wounds in the groin after vascular surgery: an early interim analysis. International Wound Journal 2013; 10(4): 377-382.

66. Monsen C, Wann-Hansson C, Wictorsson C, Acosta S. Vacuum-assisted wound closure versus alginate for the treatment of deep perivascular wound infections in the groin after vascular surgery. Journal of Vascular Surgery 2014; 59(1): 145-151.
67. Monsen C, Acosta S, Mani K, Wann-Hansson C. A randomised study of NPWT closure versus alginate dressings in peri-vascular groin infections: quality of life, pain and cost. Journal of Wound Care 2015; 24(6): 252, 254256, 258-260.

68. Arti $\mathrm{H}$, Khorami M, Ebrahimi-Nejad V. Comparison of negative pressure wound therapy (NPWT) \& conventional wound dressings in the open fracture wounds. Pak J Med Sci 2016; 32(1): 65-69.

69. Vice Chancellor for Research Technology Development, Ahvaz Jondishapur University of Medical Sciences. Comparison of suction sponge and conventional wound dressings in the open fracture wounds [online]. In: Iranian Registry of Clinical Trials. [Zugriff: 28.08.2017]. URL: http://www.irct.ir/ searchresult.php?id=14190\&number $=8$.

70. Ashby RL, Dumville JC, Soares MO, McGinnis E, Stubbs N, Torgerson DJ et al A pilot randomised controlled trial of negative pressure wound therapy to treat grade III/IV pressure ulcers [ISRCTN69032034]. Trials 2012; 13: 119.

71. University of York. Topical negative pressure (TNP) therapy to treat grade III/ IV pressure ulcers [online]. In: ISRCTN Registry. 21.08.2015 [Zugriff: 30.08 2017]. URL: http://isrctn.com/ISRCTN69032034.

72. Banasiewicz T, Bobkiewicz A, Borejsza-Wysocki M, Biczysko M, Ratajczak A, Malinger $S$ et al. Portable VAC therapy improve the results of the treatment of the pilonidal sinus: randomized prospective study. Polski Przeglad Chirurgiczny 2013; 85(7): 371-376.

73. Bee TK, Croce MA, Magnotti LJ, Zarzaur BL, Maish GO 3rd, Minard G et al. Temporary abdominal closure techniques: a prospective randomized trial comparing polyglactin 910 mesh and vacuum-assisted closure. J Trauma 2008; 65(2): 337-344.

74. Biter LU, Beck GMN, Mannaerts GHH, Stok MM, Van der Ham AC, Grotenhuis BA. The use of negative-pressure wound therapy in pilonidal sinus disease: a randomized controlled trial comparing negative-pressure wound therapy versus standard open wound care after surgical excision. Diseases of the Colon and Rectum 2014; 57(12): 1406-1411.

75. Joos A. Einsatz der Vakuumtherapie beim Sinus pilonidalis: eine randomisierte kontrollierte Studie zum Vergleich der UnterdruckWundtherapie versus Standard-Wundversorgung nach chirurgischer Exzision. Coloproctology 2015; 37(3): 210-212.

76. Braakenburg A, Obdeijn MC, Feitz R, Van Rooij IALM, Van Griethuysen AJ, Klinkenbijl JHG. The clinical efficacy and cost effectiveness of the vacuumassisted closure technique in the management of acute and chronic wounds: a randomized controlled trial. Plast Reconstr Surg 2006; 118(2): 390-397.

77. Smith \& Nephew. A 100 subjects, prospective, randomised, clinical evaluation comparing clinical and health economic outcomes between subjects treated with either PICO ( 4 weeks treatment then standard care up to 12 weeks total) or standard care dressings over 12 weeks of treatment: study CE/044/PIC; clinical evaluation report; volume 1 [unveröffentlicht]. 2016.

78. Smith \& Nephew. Trial of PICO versus standard care in chronic and subacute wounds: study details [online]. In: ClinicalTrials.gov. 13.02.2018 [Zugriff: 06.11.2018]. URL: https://ClinicalTrials.gov/show/NCT02458859.

79. Smith \& Nephew. A 100 subjects, prospective, randomised, clinical evaluation comparing clinical and health economic outcomes between subjects treated with either PICO (4 weeks treatment then standard care up to 12 weeks total) or standard care dressings over 12 weeks of treatment: study CE/044/PIC; clinical evaluation report; volume 2 [unveröffentlicht]. 2016.

80. Smith \& Nephew. A 100 subjects, prospective, randomised, clinical evaluation comparing clinical and health economic outcomes between subjects treated with either PICO (4 weeks treatment then standard care up to 12 weeks total) or standard care dressings over 12 weeks of treatment: study CE/044/PIC; clinical evaluation report; volume 3 [unveröffentlicht]. 2016.

81. Smith \& Nephew. A 100 subjects, prospective, randomised, clinical evaluation comparing clinical and health economic outcomes between subjects treated with either PICO (4 weeks treatment then standard care up to 12 weeks total) or standard care dressings over 12 weeks of treatment: study CE/044/PIC; clinical evaluation report; volume 4 [unveröffentlicht]. 2016

82. Smith \& Nephew. A 100 patient, prospective, randomised, clinical evaluatian comparing clinical and health economic outcomes between patients with chronic or sub-acute wounds treated with either PICO or standard care dressings and a qualitative study ta explore the experiences af patients receiving PICO with particular emphasis an concordance: study CE/044/PIC; clinical study protocol; amendement 1, 2, 3, 4 [unveröffentlicht]. 2013. 
83. Chiang N, Rodda OA, Sleigh J, Vasudevan T. Effects of topical negative pressure therapy on tissue oxygenation and wound healing in vascular foot wounds. J Vasc Surg 2017; 66(2): 564-571.

84. Correa JC, Mejia DA, Duque N, J MM, Uribe CM. Managing the open abdomen: negative pressure closure versus mesh-mediated fascial traction closure; a randomized trial. Hernia 2016; 20(2): 221-229.

85. Universidad de Antioquia. Open abdomen: vacuum pack versus sylo bag and mesh protocol; full text view [online]. In: ClinicalTrials.gov. 26.10.2016 [Zugriff: 30.08.2017]. URL: https://ClinicalTrials.gov/show/NCT01864590.

86. Dalla Paola L, Carone A, Ricci S, Russo A, Caccacci T, Ninkovic S. Use of vacuum assisted closure therapy in the treatment of diabetic foot wounds. Journal of Diabetic Foot Complications 2010; 2(2): 33-44.

87. De Laat EHEW, Van den Boogaard MHWA, Spauwen PHM, Van Kuppevelt DHJM, Van Goor H, Schoonhoven L. Faster wound healing with topical negative pressure therapy in difficult-to-heal wounds: a prospective randomized controlled trial. Annals of Plastic Surgery 2011; 67(6): 626-631.

88. Radboud University Medical Center Nijmegen. Faster wound healing with topical negative pressure therapy in difficult to heal wounds [online]. In: ISRCTN Registry. 24.07.2009 [Zugriff: 30.08.2017]. URL: http://isrctn.com/ ISRCTN36051617.

89. Private Universität Witten/Herdecke. Randomisiert, kontrollierte klinische Studie zur Untersuchung der Wirksamkeit und des klinischen Nutzens der Unterdruck-Wundtherapie zur Behandlung von Diabetischen Fußwunden im Vergleich zur Standard-Wundtherapie; study DiaFu; Abschlussbericht [unveröffentlicht]. 2018.

90. Seidel D, Mathes T, Lefering R, Storck M, Lawall H, Neugebauer EAM. Negative pressure wound therapy versus standard wound care in chronic diabetic foot wounds: study protocol for a randomized controlled trial. Trials 2014; 15: 334.

91. Seidel D, Mathes T, Affupper-Fink M, Lefering R, Neugebauer EAM. Studie zum Nutzennachweis der Unterdruck-Wundtherapie: innerhalb des Behandiungskonzeptes der diabetischen Fußwunde im Auftrag der gesetzlichen Krankenkassen. Vasomed 2012; 24(5): 246-251.

92. University of Witten/Herdecke. Treatment study of negative pressure wound therapy for diabetic foot wounds (DiaFu): full text view [online]. In: ClinicalTrials.gov. 18.08.2016 [Zugriff: 30.08.2017]. URL: https://ClinicalTrials. gov/show/NCT01480362.

93. Private Universität Witten/Herdecke. Randomisiert, kontrollierte klinische Studie zur Untersuchung der Wirksamkeit und des klinischen Nutzens der Unterdruck-Wundtherapie zur Behandlung von diabetischen Fußwunden im Vergleich zur Standardwundtherapie: ein Studienprojekt mit pragmatischem Ansatz zur Evaluation der Unterdruck-Wundtherapie innerhalb der medizinischen Behandlungssektoren in Deutschland [online]. In: Deutsches Register Klinischer Studien. 22.11.2011 [Zugriff: 30.08.2017]. URL: http://www. drks.de/DRKS00003347.

94. Private Universität Witten/Herdecke. Randomisiert, kontrollierte klinische Studie zur Untersuchung der Wirksamkeit und des klinischen Nutzens der Unterdruck-Wundtherapie zur Behandlung von Diabetischen Fußwunden im Vergleich zur Standard-Wundtherapie: study DiaFu; A73-03 Tabellen; Loss to Follow-UP und Begründungen [unveröffentlicht]. 2018.

95. Private Universität Witten/Herdecke. Randomisiert, kontrollierte klinische Studie zur Untersuchung der Wirksamkeit und des klinischen Nutzens der Unterdruck-Wundtherapie zur Behandlung von Diabetischen Fußwunden im Vergleich zur Standardwundtherapie: study Diafu; Studienprotokoll; Version 3.0 [unveröffentlicht]. 2013.

96. Gupta K, Mundada A, Patil A. Comparison of vacuum assisted closure therapy with standard wound therapy for open musculoskeletal injuries. International Journal of Recent Trends in Science and Technology 2013; 9(2): 168-170.

97. Hu KX, Zhang HW, Zhou F, Yao G, Shi JP, Cheng Z et al. Observation on the therapeutic effects of negative-pressure wound therapy on the treatment of complicated and refractory wounds [Chinesisch]. Zhonghua Shao Shang Za Zhi 2009; 25(4): 249-252.

98. Huang WS, Hsieh SC, Hsieh CS, Schoung JY, Huang T. Use of vacuumassisted wound closure to manage limb wounds in patients suffering from acute necrotizing fasciitis. Asian Journal of Surgery 2006; 29(3): 135-139.

99. Jayakumar M, Ajai P. A comparative study between primary vacuum assisted closure and conventional sterile dressing in treatment of soft tissue injuries associated with severe open fractures of both bones leg. Kerala Journal of Orthopaedics 2013; 26(1): 8-12

100. Kakagia D, Karadimas EJ, Drosos G, Ververidis A, Trypsiannis G, Verettas D. Wound closure of leg fasciotomy: comparison of vacuum-assisted closure versus shoelace technique; a randomised study. Injury 2014; 45(5): 890-893.
101. Karatepe O, Eken I, Acet E, Unal O, Mert M, Koc B et al. Vacuum assisted closure improves the quality of life in patients with diabetic foot. Acta Chirurgica Belgica 2011; 111(5): 298-302.

102. Leclercq A, Labeille B, Perrot JL, Vercherin P, Cambazard F. Skin graft secured by VAC (vacuum-assisted closure) therapy in chronic leg ulcers: a controlled randomized study. Annales de Dermatologie et de Venereologie 2016; 143(1): 3-8.

103. Liao Q, Xu J, Weng XJ, Zhong D, Liu Z, Wang C. Effectiveness of vacuum sealing drainage combined with anti-taken skin graft for primary closing of open amputation wound [Chinesisch]. Zhongguo Xiu Fu Chong Jian Wai Ke Za Zhi 2012; 26(5): 558-562.

104. Llanos S, Danilla S, Barraza C, Armijo E, Pineros JL, Quintas M et al. Effectiveness of negative pressure closure in the integration of split thickness skin grafts: a randomized, double-masked, controlled trial. Ann Surg 2006; 244(5): 700-705.

105. Mody GN, Nirmal IA, Duraisamy S, Perakath B. A blinded, prospective, randomized controlled trial of topical negative pressure wound closure in India. Ostomy Wound Manage 2008; 54(12): 36-46.

106. Mohsin M, Zargar HR, Wani AH, Zaroo Ml, Baba PUF, Bashir SA et al. Role of customised negative-pressure wound therapy in the integration of split-thickness skin grafts: a randomised control study. Indian J Plast Surg 2017; 50(1): 43-49.

107. Moisidis E, Heath T, Boorer C, Ho K, Deva AK. A prospective, blinded, randomized, controlled clinical trial of topical negative pressure use in skin grafting. Plast Reconstr Surg 2004; 114(4): 917-922.

108. Moues CM, Vos MC, Van den Bemd GJCM, Stijnen T, Hovius SER. Bacterial load in relation to vacuum-assisted closure wound therapy: a prospective randomized trial. Wound Repair Regen 2004; 12(1): 11-17.

109. Moues CM, Van den Bemd GJCM, Heule F, Hovius SER. Comparing conventional gauze therapy to vacuum-assisted closure wound therapy: a prospective randomised trial. J Plast Reconstr Aesthet Surg 2007; 60(6): 672-681.

110. Moues CM, Van Toorenenbergen AW, Heule F, Hop WC, Hovius SER. The role of topical negative pressure in wound repair: expression of biochemical markers in wound fluid during wound healing. Wound Repair and Regeneration 2008; 16(4): 488-494.

111. Nain PS, Uppal SK, Garg R, Bajaj K, Garg S. Role of negative pressure wound therapy in healing of diabetic foot ulcers. Journal of Surgical Technique and Case Report 2011; 3(1): 17-22.

112. Novinscak T, Zvorc M, Trojko S, Jozinovic E, Filipovic M, Grudic R. Comparison of cost-benefit ofthe three methods of diabetic ulcer treatment: dry, moistand negative pressure [Kroatisch]. Acta Medica Croatica 2010; 64(Suppl 1): 113-115.

113. Perez D, Bramkamp M, Exe C, Von Ruden C, Ziegler A. Modern wound care for the poor: a randomized clinical trial comparing the vacuum system with conventional saline-soaked gauze dressings. American Journal of Surgery 2010; 199(1): 14-20.

114. Rencüzogullari A, Dalci K, Eray IC, Yalav O, Okoh AK, Akcam T et al. Comparison of early surgical alternatives in the management of open abdomen: a randomized controlled study. Ulus Travma Acil Cerrahi Derg 2015; 21(3): 168-174

115. Saaiq M, Hameed-Ud-Din, Khan MI, Chaudhery SM. Vacuum-assisted closure therapy as a pretreatment for split thickness skin grafts. J Coll Physicians Surg Pak 2010; 20(10): 675-679.

116. Shen CA, Chai JK, Tuo XY, Cai JH, Li DJ, Zhang L et al. Efficacy observation on application of negative pressure therapy in the treatment of superficial partial-thickness scald wound in children [Chinesisch]. Zhonghua Shao Shang Za Zhi 2013; 29(1): 14-17.

117. Sibin JP, Binoj R, Jose FC. Vacuum assisted closure in grade III open tibial fractures. Indian J Appl Res 2017; 7(4): 254-256.

118. Sinha K, Chauhan VD, Maheshwari R, Chauhan N, Rajan M, Agrawal A. Vacuum assisted closure therapy versus standard wound therapy for open musculoskeletal injuries. Adv Orthop 2013; 2013: 245940.

119. Arundel C, Fairhurst C, Corbacho-Martin B, Buckley H, Clarke E, Cullum N et al. Pilot feasibility randomized clinical trial of negative-pressure wound therapy versus usual care in patients with surgical wounds healing by secondary intention. BJS Open 2018; 2(3): 99-111.

120. Arundel C, Buckley H, Clarke E, Cullum N, Dixon S, Dumville J et al. Negative pressure wound therapy versus usual care for surgical wounds healing by secondary intention (SWHSI trial): study protocol for a randomised controlled pilot trial. Trials 2016; 17(1): 535.

121. Hull and East Yorkshire Hospitals NHS Trust. Surgical wounds healing by secondary intention (SWHSI): a pilot randomised controlled trial comparing 
negative pressure wound therapy and usual care for surgical wounds healing by secondary intention [online]. In: ISRCTN Registry. 10.11.2016 [Zugriff: 30.08.2017]. URL: http://isrctn.com/ISRCTN12761776.

122. Bloemen MCT, Van der Wal MBA, Verhaegen PD, Nieuwenhuis MK, Van Baar ME, Van Zuijlen PPM et al. Clinical effectiveness of dermal substitution in burns by topical negative pressure: a multicenter randomized controlled trial. Wound Repair and Regeneration 2012; 20(6): 797-805.

123. Hop MJ, Bloemen MCT, Van Baar ME, Nieuwenhuis MK, Van Zuijlen PPM, Polinder $\mathrm{S}$ et al. Cost study of dermal substitutes and topical negative pressure in the surgical treatment of burns. Burns 2014; 40(3): 388-396.

124. Association of Dutch Burn Centres. Dermal substitute and topical negative pressure in burns (VAC-M): full text view [online]. In: ClinicalTrials.gov. 23.03. 2011 [Zugriff: 30.08.2017]. URL: https://ClinicalTrials.gov/show/NCT00548314.

125. $\mathrm{KCl}$. A randomized, controoled multicenter trial of vaccuum assisted cloaure therapy in the treatment and blinded evaluation of pressure ulcers: study VAC2001-01; final status report [unveröffentlicht]. 2007.

126. $\mathrm{KCl}$. Stellungnahme von $\mathrm{KCl}$ zum Vorbericht Vakuumversiegelungstherapie von Wunden mit intendierter sekundärer Wundheilung N17-01A. [Demnächst verfügbar unter: https://www.iqwig.de/de/projekte-ergebnisse/ projekte/nichtmedikamentoese-verfahren/n-projekte/n17-01avakuumversiegelungstherapie-von-wunden-mit-intendierter-sekundaererwundheilung.9654.html\#documents im Dokument "Dokumentation der Anhörung zum Vorbericht"].

127. KCI USA. A randomized, controlled multicenter trial of vacuum assisted closure therapy in the treatment and blinded evaluation of pressure ulcers: study VAC2001-01; study protocol amendment no. 1 [unveröffentlicht]. 2001.

128. $\mathrm{KCl}$. A randomized, controlled multicenter trial of vacuum assisted closure therapy in the treatment and blinded evaluation of venous stasis ulcers: study VAC2001-02; final status report [unveröffentlicht]. 2005.

129. $\mathrm{KCl}$. A randomized, controlled multicenter trial of vacuum assisted closure therapy in the treatment and blinded evaluation of venous stasis ulcers: study VAC2001-02; clinical research protocol [unveröffentlicht]. 2003.

130. KCl. Rohdaten zur Studie VAC 2001-03 [unveröffentlicht].

131. Lantis JC 2nd. Venous leg ulcer management: treatment options and V.A.C. therapy [Präsentationsfolien]. 2011.

132. Stannard JP, Volgas DA, Stewart R, McGwin G Jr, Alonso JE. Negative pressure wound therapy after severe open fractures: a prospective randomized study. Journal of Orthopaedic Trauma 2009; 23(8): 552-557.

133. University of Alabama at Birmingham. Vacuum assisted closure as a treatment for open fractures (VAC-OF): full text view [online]. In: ClinicalTrials.gov. 05.08.2013 [Zugriff: 30.08.2017]. URL: https://ClinicalTrials. gov/show/NCT00582361

134. University of Alabama at Birmingham. Vacuum assisted closure as a treatment for open fractures (VAC-OF): study results [online]. In: ClinicalTrials. gov. 05.08.2013 [Zugriff: 30.08.2017]. URL: https://clinicaltrials.gov/ct2/show/ results/NCT00582361.

135. KCI USA. A randomized, controlled multicenter trial of vacuum assisted closure therapy in the treatment and blinded evaluation of amputation wounds of the diabteic foot: study VAC 2001-07; clinical study report [unveröffentlicht]. 2005

136. Armstrong DG, Lavery LA. Negative pressure wound therapy after partial diabetic foot amputation: a multicentre, randomised controlled trial. Lancet 2005; 366(9498): 1704-1710.

137. Apelqvist J, Armstrong DG, Lavery LA, Boulton AJM. Resource utilization and economic costs of care based on a randomized trial of vacuum-assisted closure therapy in the treatment of diabetic foot wounds. American Journal of Surgery 2008; 195(6): 782-788.

138. Lavery LA, Barnes SA, Keith MS, Seaman JJ, Armstrong DG. Prediction of healing for postoperative diabetic foot wounds based on early wound area progression. Diabetes Care 2008; 31(1): 26-29.

139. Armstrong DG, Lavery LA, Boulton AJM. Negative pressure wound therapy via vacuum-assisted closure following partial foot amputation: what is the role of wound chronicity? International Wound Journal 2007; 4(1): 79-86.

140. KCI USA. Trial of vacuum assisted closure therapy in amputation wounds of the diabetic foot: full text view [online]. In: ClinicalTrials.gov. 19.07.2006 [Zugriff: 30.08.2017]. URL: https://ClinicalTrials.gov/show/NCT00224796.

141. KCI USA. A randomized, controlled multicenter trial of vacuum assisted closure therapy in the treatment and blinded evaluation of amputation wounds of the diabetic foot: study VAC2001-07; clinical research protocol: amendment \# 2 [unveröffentlicht]. 2004
142. KCI USA. A randomized, controlled multicenter trial of vacuum assisted closure therapy in the treatment and blinded evaluation of diabetic foot ulcers: study VAC 2001-08; clinical study report [unveröffentlicht]. 2005.

143. Driver VR, Blume PA. Evaluation of wound care and health-care use costs in patients with diabetic foot ulcers treated with negative pressure wound therapy versus advanced moist wound therapy. Journal of the American Podiatric Medical Association 2014; 104(2): 147-153.

144. Blume PA, Walters J, Payne W, Ayala J, Lantis J. Comparison of negative pressure wound therapy using vacuum-assisted closure with advanced moist wound therapy in the treatment of diabetic foot ulcers: a multicenter randomized controlled trial. Diabetes Care 2008; 31(4): 631-636.

145. Blume PA. Comparison of negative pressure wound therapy using vacuum-assisted closure with advanced moist wound therapy in the treatment of diabetic foot ulcers: a multicenter randomized controlled trial. Diabetes Care 2008; 31(10): e77.

146. Hemkens LG, Waltering A. Comparison of negative pressure wound therapy using vacuum-assisted closure with advanced moist wound therapy in the treatment of diabetic foot ulcers: a multicenter randomized controlled trial. Diabetes Care 2008; 31(10): e76.

147. KCI USA. Randomized, controlled multicenter trial of Vacuum Assisted Closure Therapy in diabetic foot ulcers: study details [online]. In: ClinicalTrials.gov. 23.01.2018 [Zugriff: 06.11.2018]. URL: https://ClinicalTrials. gov/show/NCT00432965.

148. KCI USA. Randomized, controlled multicenter trial of vacuum assisted closure therapy in diabetic foot ulcers: study results [online]. In: ClinicalTrials. gov. 23.01.2018 [Zugriff: 06.11.2018]. URL: https:/clinicaltrials.gov/ct2/show/ results/NCT00432965.

149. KCI USA. A randomized, controlled multicenter trial of vacuum assisted closure therapy in the treatment and blinded evaluation of diabetic foot ulcers: study VAC2001-08; study protocol amendment no. 1 [unveröffentlicht]. 2002

150. KCI USA. A randomized, controlled multicenter trial of vacuum assisted closure therapy in the treatment and blinded evaluation of open chest wounds: study VAC2002-09; final status report [unveröffentlicht]. 2006.

151. KCI USA. A randomized, controlled multicenter trial of vacuum assisted closure therapy in the treatment and blinded evaluation of open chest wounds: study VAC2002-09; study protocol amendment no. 1 [unveröffentlicht]. 2002.

152. $\mathrm{KCl}$ USA. A randomized, controlled multicenter trial of vacuum assisted closure therapy in the treatment and blinded evaluation of open abdominal wounds: study VAC2002-10; final status report [unveröffentlicht]. 2006.

153. KCI USA. A randomized, controlled multicenter trial of vacuum assisted closure therapy in the treatment and blinded evaluation of open abdominal wounds: study VAC2002-10; study protocol amendment no. 1 [unveröffentlicht]. 2002.

154. Virani SR, Dahapute AA, Bava SS, Muni SR. Impact of negative pressure wound therapy on open diaphyseal tibial fractures: a prospective randomized trial. J Clin Orthop Trauma 2016; 7(4): 256-259.

155. Vuerstaek JDD, Vainas T, Wuite J, Nelemans P, Neumann MHA, Veraart JCJM. State-of-the-art treatment of chronic leg ulcers: a randomized controlled trial comparing vacuum-assisted closure (V.A.C.) with modern wound dressings. J Vasc Surg 2006; 44(5): 1029-1037.

156. Ahmed M, Soskova T, Williams DT. Regarding "state-of-the-art treatment of chronic leg ulcers: a randomized controlled trial comparing vacuum-assisted closure (V.A.C.) with modern wound dressings". Journal of Vascular Surgery 2007; 46(3): 614-615.

157. Maastricht University Medical Center. A randomised controlled trial comparing vacuum assisted closure (V.A.C.) with modern wound dressings: full text view [online]. In: ClinicalTrials.gov. 12.01.2017 [Zugriff: 30.08.2017]. URL: https://ClinicalTrials.gov/show/NCT00243620.

158. Costa ML, Achten J, Bruce J, Tutton E, Petrou S, Lamb SE et al. Effect of negative pressure wound therapy vs standard wound management on 12month disability among adults with severe open fracture of the lower limb: the WOLLF randomized clinical trial. JAMA 2018; 319(22): 2280-2288.

159. Achten J, Parsons NR, Bruce J, Petrou S, Tutton E, Willett K et al. Protocol for a randomised controlled trial of standard wound management versus negative pressure wound therapy in the treatment of adult patients with an open fracture of the lower limb: UK wound management of open lower limb fractures (UK WOLFF). BMJ Open 2015; 5(9): e009087.

160. Tutton E, Achten J, Lamb SE, Willett K, Costa ML. Participation in a trial in the emergency situation: a qualitative study of patient experience in the UK WOLLF trial. Trials 2018; 19(1): 328. 
161. University of Warwick. Wound management of open lower limb fractures (WOLLF) [online]. In: ISRCTN Registry. 02.07.2018 [Zugriff: 26.07.2018]. URL: http://isrctn.com/ISRCTN33756652.

162. Xu XP, Cai XH, Zhang L, Li XH, Liu ZH, Pan Y et al. Clinical efficacy of vacuumsealing drainage in treatment of fournier's gangrene [Chinesisch]. Shi Jie Hua Ren Xiao Hua Za Zhi 2015; 23(2): 348-352.

163. Canadian Agency for Drugs and Technologies in Health. Negative pressure wound therapy for managing diabetic foot ulcers: a review of the clinical effectiveness, cost-effectiveness, and guidelines [online]. 28.08.2014 [Zugriff: 14.11.2017]. URL: https://www.cadth.ca/media/pdf/htis/dec-2014/RC0579001\%20Diabetic\%20Foot\%20Ulcers\%20Final.pdf.

164. Dumville JC, Hinchliffe RJ, Cullum N, Game F, Stubbs N, Sweeting M et al. Negative pressure wound therapy for treating foot wounds in people with diabetes mellitus. Cochrane Database of Systematic Reviews 2013; (1): CD010318.

165. Dumville JC, Land L, Evans D, Peinemann F. Negative pressure wound therapy for treating leg ulcers. Cochrane Database of Systematic Reviews 2015; (7): CD011354.

166. Liu S, He CZ, Cai YT, Xing QP, Guo YZ, Chen ZL et al. Evaluation of negativepressure wound therapy for patients with diabetic foot ulcers: systematic review and meta-analysis. Ther Clin Risk Manag 2017; 13: 533-544.

167. Malaysian Health Technology Assessment. Disposable negative pressure wound therapy: 026/2013 [online]. [Zugriff: 14.11.2017]. URL: http://www. moh.gov.my/index.php/database_stores/attach_download/347/229.

168. Pan A, De Angelis G, Nicastri E, Sganga G, Tacconelli E. Topical negative pressure to treat surgical site infections, with a focus on post-sternotomy infections: a systematic review and meta-analysis. Infection 2013; 41(6): 1129-1135.

169. Walker M, Kralik D, Porritt K. Fasciotomy wounds associated with acute compartment syndrome: a systematic review of effective treatment. JBI Database System Rev Implement Rep 2014; 12(1): 101-175.

170. Wang R, Feng Y, Di B. Comparisons of negative pressure wound therapy and ultrasonic debridement for diabetic foot ulcers: a network metaanalysis. Int J Clin Exp Med 2015; 8(8): 12548-12556.

171. Zhang J, Hu ZC, Chen D, Guo D, Zhu JY, Tang B. Effectiveness and safety of negative-pressure wound therapy for diabetic foot ulcers: a meta-analysis. Plastic and Reconstructive Surgery 2014; 134(1): 141-151.

172. Liu X, Zhang H, Cen S, Huang F. Negative pressure wound therapy versus conventional wound dressings in treatment of open fractures: a systematic review and meta-analysis. Int J Surg 2018; 53: 72-79.

173. Dumville JC, Webster J, Evans D, Land L. Negative pressure wound therapy for treating pressure ulcers. Cochrane Database of Systematic Reviews 2015; (5): CD011334

174. Reddy M. Pressure ulcers: treatment. BMJ Clin Evid 2015; 2015: pii: 1901.

175. Iheozor-Ejiofor Z, Newton K, Dumville JC, Costa ML, Norman G, Bruce J. Negative pressure wound therapy for open traumatic wounds. Cochrane Database Syst Rev 2018; (7): CD012522.

176. Cirocchi R, Birindelli A, Biffl WL, Mutafchiyski V, Popivanov G, Chiara O et al. What is the effectiveness of the negative pressure wound therapy (NPWT) in patients treated with open abdomen technique? A systematic review and meta-analysis. J Trauma Acute Care Surg 2016; 81(3): 575-584.

177. Dumville JC, Owens GL, Crosbie EJ, Peinemann F, Liu Z. Negative pressure wound therapy for treating surgical wounds healing by secondary intention. Cochrane Database of Systematic Reviews 2015; (6): CD01 1278.

178. Janssen AHJ, Mommers EHH, Notter J, De Vries Reilingh TS, Wegdam JA. Negative pressure wound therapy versus standard wound care on quality of life: a systematic review. Journal of Wound Care 2016; 25(3): 154, 156-159.

179. Rhee SM, Valle MF, Wilson LM, Lazarus G, Zenilman JM, Robinson KA. Negative pressure wound therapy technologies for chronic wound care in the home setting: technology assessment report [online]. 15.09.2014 [Zugriff: 21.11.2017]. URL: https://www.ncbi.nlm.nih.gov/books/NBK285361/ pdf/Bookshelf_NBK285361.pdf.

180. Yin Y, Zhang R, Li S, Guo J, Hou Z, Zhang Y. Negative-pressure therapy versus conventional therapy on split-thickness skin graft: a systematic review and meta-analysis. International Journal Of Surgery 2018; 50: 43-48.

181. Song F, Parekh S, Hooper L, Loke YK, Ryder J, Sutton AJ et al. Dissemination and publication of research findings: an updated review of related biases. Health Technology Assessment 2010; 14(8): 1-193.

\section{Publisher's Note}

Springer Nature remains neutral with regard to jurisdictional claims in published maps and institutional affiliations.

Ready to submit your research? Choose BMC and benefit from:

- fast, convenient online submission

- thorough peer review by experienced researchers in your field

- rapid publication on acceptance

- support for research data, including large and complex data types

- gold Open Access which fosters wider collaboration and increased citations

- maximum visibility for your research: over $100 \mathrm{M}$ website views per year

At BMC, research is always in progress.

Learn more biomedcentral.com/submissions 